\title{
Large cardinals and covering numbers
}

by

Pierre Matet (Caen)

\begin{abstract}
The paper is concerned with the computation of covering numbers in the presence of large cardinals. In particular, we revisit Solovay's result that the Singular Cardinal Hypothesis holds above a strongly compact cardinal.
\end{abstract}

0. Introduction. The Generalized Continuum Hypothesis (GCH) asserts that $2^{\rho}=\rho^{+}$for every infinite cardinal $\rho$, and the Singular Cardinal Hypothesis $(\mathrm{SCH})$ that $\nu^{\operatorname{cf}(\nu)}=\nu^{+} \cdot 2^{\operatorname{cf}(\nu)}$ for any singular cardinal $\nu$. One of the many equivalent reformulations of GCH states that $\left|P_{\kappa}(\lambda)\right| \leq \lambda^{+}$for every regular uncountable cardinal $\kappa$ and every cardinal $\lambda \geq \kappa$. Similarly, SCH can be rephrased as the assertion that $\left|P_{\kappa}(\lambda)\right| \leq 2^{<\kappa} \cdot \lambda^{+}$for all $\kappa$ and $\lambda$ as above. Now since $\left|P_{\kappa}(\lambda)\right|=2^{<\kappa} \cdot u(\kappa, \lambda)$ (where $u(\kappa, \lambda)$ denotes the least cardinality of a cofinal subset of $\left(P_{\kappa}(\lambda), \subseteq\right)$ ), it is natural to consider the statement that $u(\kappa, \lambda) \leq \lambda^{+}$for all $\kappa$ and $\lambda$ as above. This assertion is equivalent to Shelah's Strong Hypothesis (SSH).

What is the relative status of these three hypotheses? Clearly GCH implies SSH, which in turn implies SCH. Neither implication can be reversed. Adding $\aleph_{2}$ Cohen reals to a model of GCH will yield a model of " $\neg$ GCH + SSH". And, as Moti Gitik pointed out to the author, one obtains a model of " $u\left(\omega_{1}, \omega_{\omega}\right)>\omega_{\omega}^{+}$(and hence $\left.\neg \mathrm{SSH}\right)+\mathrm{SCH}$ " by adding $\aleph_{\omega+1}$ Cohen reals to a model of "for every infinite cardinal $\nu, 2^{\nu}$ equals $\nu^{++}$if $\nu=\omega_{\omega}$, and $\nu^{+}$otherwise". Unlike that of GCH, the failure of SSH has large cardinal strength. In fact, if the covering lemma holds with respect to some inner model satisfying GCH, then SSH holds. Gitik [10] established that the failure of $\mathrm{SCH}$ is equiconsistent with the existence of a cardinal $\nu$ with $o(\nu)=\nu^{++}$. It is not known whether the failure of SSH is equiconsistent with that of SCH. We will show (Proposition 3.8) that if SCH holds, then $u(\kappa, \lambda) \leq \lambda^{+}$for every regular uncountable cardinal $\kappa$ and every cardinal $\lambda \geq \kappa \cdot \tau$, where $\tau$ is the least uncountable strong limit cardinal. 
The starting point of this paper is Solovay's epochal result [33] that SCH holds above a strongly compact cardinal. More precisely, Solovay proved the following local result: Let $\kappa$ be a regular uncountable cardinal. Suppose $\kappa$ is $\tau$-compact, where $\tau \geq \kappa$ is a regular cardinal. Then $\left|P_{\kappa}(\tau)\right|=\tau$, and in fact $\tau^{\rho}=\tau$ for every regular infinite cardinal $\rho$ with $2^{\rho}<\tau$. Now using Silver's Theorem [31] and the fact that if $\kappa$ is $\sigma$-compact for some cardinal $\sigma>\kappa$ with $\operatorname{cf}(\sigma)<\kappa$, then $\kappa$ is $\sigma^{+}$-compact, it easily follows that if $\lambda>\kappa$ is a singular cardinal such that $\kappa$ is $\lambda$-compact, then $\lambda^{\operatorname{cf}(\lambda)}=\lambda^{+} \cdot 2^{\operatorname{cf}(\lambda)}$. Can one improve this result? First look at the conclusion. Consider for instance the case when $\lambda=\kappa^{+\kappa}<2^{\kappa}$. Then of course $\lambda^{\kappa}=2^{\kappa}$ but this is not very informative (and does not make use of the $\lambda$-compactness of $\kappa$ !). One way to make progress is to switch from the computation of $\lambda^{\kappa}$ to that of $u\left(\kappa^{+}, \lambda\right)$, and more generally to that of $u(\mu, \lambda)$ for a regular cardinal $\mu$ with $\kappa<\mu<\lambda$.

Now what about weakening the assumption of Solovay's result? There is more than one possibility since the $\lambda$-compactness of $\kappa$ can be formulated in several ways (for instance in terms of compactness properties of the infinitary language $\mathcal{L}_{\kappa \kappa}$, or in terms of existence of prime ideals on $P_{\kappa}(\lambda)$ ). Also note that the assumption can be made "weaker" in more than one sense. For example we could look for an assumption of lesser consistency strength. Or we could try to make $\kappa$ small. How small can small be? Well, as small as $\omega_{2}$, as shown by Todorcevic ([34], [35]) who proved (from a supercompact cardinal) the consistency of Rado's Conjecture (RC) and established that $\mathrm{RC}$ implies SCH. In fact it implies SSH since Todorcevic also proved that RC entails $2^{\aleph_{0}} \leq \aleph_{2}$. "Small" could also mean "smaller than $2^{\aleph_{0}}$ ". We will show (Proposition 7.3) that if there is an ideal on $P_{\kappa}(\lambda)$ that is $\rho$-saturated for some cardinal $\rho<\kappa$, where $\kappa$ is a regular uncountable cardinal and $\lambda \geq \kappa$ a cardinal, then for any regular cardinal $\mu$ with $\kappa \leq \mu \leq \lambda, u(\mu, \lambda)$ equals $\lambda$ if $\operatorname{cf}(\lambda) \geq \mu$, and $\lambda^{+}$otherwise.

Before he obtained his result on strongly compact cardinals, Solovay [33] showed that if $\kappa$ is $\lambda$-supercompact and $\lambda$ is regular, then the function $a \mapsto \bigcup a$ is injective on some set $A \subseteq P_{\kappa}(\lambda)$ such that $A \in J^{*}$ for every normal prime ideal $J$ on $P_{\kappa}(\lambda)$. Johnson [13] sharpened this result in the case when $\lambda$ is a successor cardinal. Namely, he showed that if $\kappa$ is $\lambda$-Shelah and $\lambda=\nu^{+}$, then the sup-function is injective on a set in $N S h_{\kappa, \lambda}^{*}$. Abe [3] remarked that Johnson's result remains valid in the case when $\lambda$ is a regular limit cardinal. We complete the picture by proving two companion results, one (Proposition 9.4) for the case when $\kappa \leq \operatorname{cf}(\lambda)<\lambda$ and the other (Proposition 9.6) for the case when $\operatorname{cf}(\lambda)<\kappa$.

The paper is organized as follows. In Section 1 we review basic material concerning ideals on $P_{\kappa}(\lambda)$. Section 2 lists several results of Shelah and others on covering numbers that will be needed later. In Section 3 we establish a result concerning the consequences of " $u(\mu, \lambda)>\lambda^{+}$" (and, more generally, 
of " $\operatorname{cov}(\lambda, \mu, \mu, \kappa)>\lambda^{+}$"). This will be the main tool in our subsequent investigation of the effect of large cardinals on cardinal arithmetic. Our motivation for considering $\operatorname{cov}(\lambda, \mu, \mu, \kappa)$ for uncountable $\kappa$ goes beyond the search for greater generality. Consider for instance the case when $\mu=\kappa^{+}$and $\lambda \geq 2^{\kappa}$. Then $\operatorname{cov}(\lambda, \mu, \mu, \kappa)=\lambda$ if and only if the ideal $I_{\kappa, \lambda} \mid C$ is $\kappa$-normal for some club subset $C$ of $P_{\kappa}(\lambda)$. This is proved in Section 4 .

In Section 5 we review results of Todorcevic ([34], [35]) and MagidorShelah [18] concerning the compactness properties wRC and PT and their impact on cardinal arithmetic. In Section 6 we extend work of Abe [2] concerning the consequences of the existence of a $\kappa^{+}$-saturated ideal on $P_{\kappa}(\lambda)$. In Section 7 we strengthen our assumption (requiring now the existence of an ideal that is $<\kappa$-saturated) to get a better conclusion.

In Section 8 we prove a $P_{\kappa}(\lambda)$ version of the result of Solovay [32] concerning the partition relation satisfied by a normal ideal on $\kappa$ that is $\tau$-saturated for some $\tau<\kappa$. In Section 9 we revisit Solovay's result [33] on the sup-function. Following Johnson [13], we assume Shelahness rather than supercompactness. Shelahness (or rather a weak variant of it) appears again in Section 10, where we remark that Krueger's striking result [15] that the strong compactness of $\kappa$ does not entail the stationarity of the set $\left\{a \in P_{\kappa}\left(\kappa^{+}\right):|a|>|a \cap \kappa|\right\}$ is in some sense optimal.

1. Ideals on $P_{\kappa}(\lambda)$. In this section we review basic material concerning ideals on $P_{\kappa}(\lambda)$. For a set $A$ and a cardinal $\rho$, we let $P_{\rho}(A)=\{a \subseteq A$ : $|a|<\rho\}$.

Let $\kappa$ be a regular uncountable cardinal, and $\lambda \geq \kappa$ be a cardinal.

$I_{\kappa, \lambda}$ denotes the collection of all $A \subseteq P_{\kappa}(\lambda)$ such that $\{a \in A: b \subseteq a\}=\emptyset$ for some $b \in P_{\kappa}(\lambda)$. An ideal on $P_{\kappa}(\lambda)$ is a collection $J$ of subsets of $P_{\kappa}(\lambda)$ such that (i) $P(A) \subseteq J$ for any $A \in J$, (ii) $\bigcup X \in J$ for every $X \in P_{\kappa}(J)$, (iii) $I_{\kappa, \lambda} \subseteq J$, and (iv) $P_{\kappa}(\lambda) \notin J$.

Let $J$ be an ideal on $P_{\kappa}(\lambda)$. We set $J^{+}=P\left(P_{\kappa}(\lambda)\right) \backslash J$ and $J^{*}=\left\{P_{\kappa}(\lambda) \backslash\right.$ $A: A \in J\}$. For $A \in J^{+}$, let $J \mid A=\left\{B \subseteq P_{\kappa}(\lambda): B \cap A \in J\right\}$. Let $\operatorname{cof}(J)$ denote the least size of any $X \subseteq J$ such that for any $A \in J$, there is $Q \in P_{\kappa}(X)$ with $A \subseteq \bigcup Q$.

Let $J$ be an ideal on $P_{\kappa}(\lambda)$, and $\delta$ be an ordinal with $\kappa \leq \delta \leq \lambda$. Then $J$ is $\delta$-normal if for every $A \in J^{+}$and every $f: A \rightarrow \delta$ such that $f(a) \in a$ for all $a \in A$, there is $B \in J^{+} \cap P(A)$ such that $f$ is constant on $B$. $N S_{\kappa, \lambda}^{\delta}$ denotes the smallest $\delta$-normal ideal on $P_{\kappa}(\lambda)$. For $f: P_{\omega}(\delta) \rightarrow P_{\kappa}(\lambda)$, let $C_{f}^{\kappa, \lambda}$ denote the set of all $a \in P_{\kappa}(\lambda)$ such that (i) $\omega \subseteq a$ and (ii) $f(e) \subseteq a$ for every $e \in P_{\omega}(a \cap \delta)$.

Lemma 1.1 (Matet-Péan-Shelah [23]). $N S_{\kappa, \lambda}^{\delta}$ is the set of all $A \subseteq P_{\kappa}(\lambda)$ such that $A \cap C_{f}^{\kappa, \lambda}=\emptyset$ for some $f: P_{\omega}(\delta) \rightarrow P_{\kappa}(\lambda)$. 
$J$ is normal if it is $\lambda$-normal. We let $N S_{\kappa, \lambda}=N S_{\kappa, \lambda}^{\lambda}$.

$J$ is seminormal if it is $\delta$-normal for every $\delta$ with $\kappa \leq \delta<\lambda . N S S_{\kappa, \lambda}$ denotes the smallest seminormal ideal on $P_{\kappa}(\lambda)$.

$J$ is strongly normal if for every $A \in J^{+}$and every $f: A \rightarrow P_{\kappa}(\lambda)$ such that $f(a) \in P_{|a \cap \kappa|}(a)$ for all $a \in A$, there is $B \in J^{+} \cap P(A)$ such that $f$ is constant on $B$.

The following is readily checked:

Lemma 1.2. Suppose $\operatorname{cf}(\lambda)<\kappa$. Then every seminormal ideal on $P_{\kappa}(\lambda)$ is normal.

Lemma 1.3 (Carr-Levinski-Pelletier [5]). Suppose $\kappa$ is a limit cardinal. Then there exists a strongly normal ideal on $P_{\kappa}(\lambda)$ if and only if $\kappa$ is Mahlo.

Assuming $\kappa$ is Mahlo, $N S_{\kappa, \lambda}^{[\lambda]<\kappa}$ denotes the smallest strongly normal ideal on $P_{\kappa}(\lambda)$.

For a cardinal $\mu, J$ is $\mu$-saturated (respectively, weakly $\mu$-saturated) if there is no $Q \subseteq J^{+}$such that (a) $|Q|=\mu$ and (b) $A \cap B \in J$ (respectively, $A \cap B=\emptyset$ ) for any two distinct members $A, B$ of $Q . J$ is prime if it is 2-saturated. $\kappa$ is $\lambda$-compact if there exists a prime ideal on $P_{\kappa}(\lambda)$, and $\lambda$-supercompact if there exists a normal prime ideal on $P_{\kappa}(\lambda) . \kappa$ is strongly compact (respectively, supercompact) if it is $\tau$-compact (respectively, $\tau$-supercompact) for every cardinal $\tau \geq \kappa$.

LEMMA 1.4.

(i) (Erdős-Tarski [9]) Let $J$ be an $\omega$-saturated ideal on $P_{\kappa}(\lambda)$. Then $J \mid A$ is prime for some $A \in J^{+}$.

(ii) (Erdős-Tarski [9]) Let $\nu$ be a singular cardinal, and $J$ be a $\nu$ saturated ideal on $P_{\kappa}(\lambda)$. Then $J$ is $\tau$-saturated for some cardinal $\tau<\nu$

(iii) Let $\nu$ be a singular cardinal, and $J$ be a weakly $\nu$-saturated ideal on $P_{\kappa}(\lambda)$. Then $J \mid A$ is weakly $\tau$-saturated for some $A \in J^{+}$and some cardinal $\tau<\nu$.

$J$ is weakly normal if (a) $J$ is weakly $\operatorname{cf}(\lambda)$-saturated, and (b) for every $A \in J^{+}$and every $f: A \rightarrow \lambda$ such that $f(a) \in a$ for all $a \in A$, there are $B \in J^{+} \cap P(A)$ and $\alpha<\lambda$ such that $f(b) \leq \alpha$ for all $b \in B$.

Let $X, Y \subseteq P\left(P_{\kappa}(\lambda)\right)$, and let $\rho$ be a cardinal. $X \rightarrow[Y]_{\rho}^{2}$ means that for every $A \in X$ and every $F: A \times A \rightarrow \rho$, there are $B \in Y \cap P(A)$ and $\xi \in \rho$ such that $F(a, b) \neq \xi$ for all $a, b \in B$ with $a \subset b$. For a cardinal $\tau \leq \rho, X \rightarrow[Y]_{\rho,<\tau}^{2}$ means that for every $A \in X$ and every $F: A \times A \rightarrow \rho$, there are $B \in Y \cap P(A)$ and $e \in P_{\tau}(\rho)$ such that $F(a, b) \in e$ for all $a, b \in B$ with $a \subset b$. 
Most of the definitions above are $P_{\kappa}(\lambda)$ versions of notions which originated in the study of $\kappa$. For the original definitions see e.g. [14]. They will not be repeated here, except for that of $\kappa \rightarrow[\kappa]_{\rho,<\tau}^{2}$ which is maybe not so widely known. $\kappa \rightarrow[\kappa]_{\rho,<\tau}^{2}$ means that for every $F: \kappa \times \kappa \rightarrow \rho$, there are $B \subseteq \kappa$ with $|B|=\kappa$ and $e \in P_{\tau}(\rho)$ such that $F(\alpha, \beta) \in e$ for all $\alpha, \beta \in B$ with $\alpha<\beta$.

Let us also recall the following:

LEMMA 1.5.

(i) (Kunen [16]) If $\kappa$ carries a $\kappa^{+}$-saturated ideal, then it is measurable in some inner model of ZFC.

(ii) (Ulam [36]) If $\kappa$ carries a $\kappa$-saturated ideal, then it is a limit cardinal.

(iii) (see [14, p. 212]) If $\kappa$ carries a $\rho$-saturated ideal for some cardinal $\rho<\kappa$, then it has the tree property.

Given sets $X$ and $Y, f: X \rightarrow Y$ and $J \subseteq P(X)$, we let $f(J)=\{A \subseteq Y$ : $\left.f^{-1}(A) \in J\right\}$.

2. Covering numbers. In this section we recall a number of results that will be needed in the sequel.

Definition. Given four cardinals $\lambda, \chi, \mu$ and $\kappa$ such that $\lambda \geq \chi \geq \mu \geq \omega$ and $\mu \geq \kappa \geq 2, \operatorname{cov}(\lambda, \chi, \mu, \kappa)$ denotes the least cardinality of any $X \subseteq P_{\chi}(\lambda)$ such that for any $a \in P_{\mu}(\lambda)$, there is $Q \in P_{\kappa}(X)$ with $a \subseteq \bigcup Q$.

Proposition 2.1 (Shelah [28, pp. 85-86]). Let $\lambda, \chi, \mu$ and $\kappa$ be four cardinals such that $\lambda \geq \chi \geq \mu \geq \omega$ and $\mu \geq \kappa \geq 2$. Then the following hold:

(i) If $\lambda=\chi$ and either $\operatorname{cf}(\lambda)<\kappa$ or $\operatorname{cf}(\lambda) \geq \mu$, then $\operatorname{cov}(\lambda, \chi, \mu, \kappa)=$ $\operatorname{cf}(\lambda)$.

(ii) If either $\lambda>\chi$, or $\lambda=\chi$ and $\kappa \leq \operatorname{cf}(\lambda)<\mu$, then $\operatorname{cov}(\lambda, \chi, \mu, \kappa) \geq \lambda$.

(iii) $\operatorname{cov}(\lambda, \chi, \mu, \kappa)=\operatorname{cov}(\lambda, \chi, \mu, \omega \cdot \kappa)$.

(iv) $\operatorname{cov}(\lambda, \chi, \mu, \kappa) \leq \operatorname{cov}\left(\lambda, \chi, \mu, \kappa^{\prime}\right)$ for every infinite cardinal $\kappa^{\prime} \leq \kappa$.

(v) $\operatorname{cov}(\lambda, \chi, \mu, \kappa) \leq \operatorname{cov}\left(\lambda, \chi, \mu^{\prime}, \kappa\right)$ for every cardinal $\mu^{\prime}$ with $\chi \geq \mu^{\prime}$ $\geq \mu$.

(vi) $\operatorname{cov}(\lambda, \chi, \mu, \kappa) \leq \operatorname{cov}\left(\lambda, \chi^{\prime}, \mu, \kappa\right)$ for every cardinal $\chi^{\prime}$ with $\chi \geq \chi^{\prime}$ $\geq \mu$.

(vii) $\operatorname{cov}(\lambda, \chi, \mu, \kappa) \leq \operatorname{cov}\left(\lambda^{\prime}, \chi, \mu, \kappa\right)$ for every cardinal $\lambda^{\prime} \geq \lambda$.

(viii) $\operatorname{cov}\left(\lambda^{+}, \chi, \mu, \kappa\right)=\lambda^{+} \cdot \operatorname{cov}(\lambda, \chi, \mu, \kappa)$.

(ix) Suppose $\lambda$ is a limit cardinal such that $\lambda>\chi$ and $\operatorname{cf}(\lambda) \geq \mu$. Then $\operatorname{cov}(\lambda, \chi, \mu, \kappa)=\bigcup_{\chi \leq \lambda^{\prime}<\lambda} \operatorname{cov}\left(\lambda^{\prime}, \chi, \mu, \kappa\right)$.

(x) If $\mu>\kappa \geq \omega$, then $\operatorname{cov}(\lambda, \chi, \mu, \kappa)=\bigcup_{\kappa \leq \rho<\mu} \operatorname{cov}\left(\lambda, \chi, \rho^{+}, \kappa\right)$. 
(xi) If $\lambda>\chi$ and $\operatorname{cf}(\lambda)<\kappa=\operatorname{cf}(\kappa)$, then

$$
\operatorname{cov}(\lambda, \chi, \mu, \kappa)=\bigcup_{\chi \leq \lambda^{\prime}<\lambda} \operatorname{cov}\left(\lambda^{\prime}, \chi, \mu, \kappa\right) .
$$

Proposition 2.2. Let $\lambda, \chi$ and $\mu$ be three uncountable cardinals such that $\lambda \geq \chi>\mu=\operatorname{cf}(\mu)>\operatorname{cf}(\chi)$. Then $\operatorname{cov}(\lambda, \chi, \mu, 2)=\operatorname{cov}(\lambda, \tau, \mu, 2)$ for some cardinal $\tau$ with $\kappa \leq \tau<\chi$.

Proof. Let $X \subseteq P_{\chi}(\lambda)$ be such that for any $a \in P_{\mu}(\lambda)$, there is $b \in X$ with $a \subseteq b$. Select an increasing sequence $\left\langle\chi_{i}: i<\operatorname{cf}(\chi)\right\rangle$ of infinite cardinals cofinal in $\chi$. For $i<\operatorname{cf}(\chi)$, put $T_{i}=\left\{d \in P_{\mu}(\lambda): \exists b \in X \cap P_{\chi_{i}}(\lambda)(d \subseteq b)\right\}$. Since $P_{\mu}(\lambda)=\bigcup_{i<\operatorname{cf}(\chi)} T_{i}$, we may find $j<\operatorname{cf}(\chi)$ such that $T_{j} \in I_{\mu, \lambda}^{+}$. Then clearly for any $a \in P_{\mu}(\lambda)$, there is $b \in T_{i}$ with $a \subseteq b$.

Proposition 2.3. Let $\lambda, \chi, \mu$ and $\kappa$ be four infinite cardinals such that $\lambda \geq \chi \geq \mu=\operatorname{cf}(\mu) \geq \kappa$. Then either $\operatorname{cf}(\operatorname{cov}(\lambda, \chi, \mu, \kappa))<\kappa$, or $\operatorname{cf}(\operatorname{cov}(\lambda, \chi, \mu, \kappa)) \geq \mu$.

Proof. Suppose toward a contradiction that $\kappa \leq \tau<\mu$, where $\tau=$ $\operatorname{cf}(\operatorname{cov}(\lambda, \chi, \mu, \kappa))$. Pick $X \subseteq P_{\chi}(\lambda)$ so that $|X|=\operatorname{cov}(\lambda, \chi, \mu, \kappa)$ and for every $a \in P_{\mu}(\lambda)$, there is $w \in P_{\kappa}(X)$ with $a \subseteq \bigcup w$. Let $X=\bigcup_{i<\tau} X_{i}$, where $\left|X_{i}\right|<|X|$ for any $i<\tau$. For $i<\tau$, select $a_{i} \in P_{\mu}(\lambda)$ so that $a_{i} \backslash \bigcup w \neq \emptyset$ for all $w \in P_{\kappa}\left(X_{i}\right)$. Now set $a=\bigcup_{i<\tau} a_{i}$. Since $a \in P_{\mu}(\lambda)$, one can find $w \in P_{\kappa}(X)$ so that $a \subseteq \bigcup w$. Then there must be $i<\tau$ such that $w \subseteq X_{i}$. Clearly, $a_{i} \subseteq \bigcup w$. Contradiction.

Corollary 2.4 (Liu [17]). Let $\lambda$ be a singular cardinal. Then $\operatorname{cov}\left(\lambda, \lambda, \kappa^{+}, \kappa\right)>\lambda$, where $\kappa=\operatorname{cf}(\lambda)$.

Proposition 2.5 (Shelah [28, Remark 6.6.A p. 101]). Let $\lambda$ be a singular cardinal and let $\kappa=\operatorname{cf}(\lambda)$. Then $\operatorname{cov}\left(\lambda, \lambda, \kappa^{+}, \kappa\right)>\lambda^{+}$if and only if $\operatorname{cov}\left(\lambda, \lambda, \kappa^{+}, 2\right)>\lambda^{+}$.

Hugh Woodin, starting from a hypermeasurability hypothesis, constructed a model where $2^{\nu}=\nu^{++}$for every infinite cardinal $\nu$. It is simple to see that in this model for every singular cardinal $\lambda, \operatorname{cov}\left(\lambda, \lambda, \kappa^{+}, 2\right)=\lambda^{++}$, where $\kappa=\operatorname{cf}(\lambda)$.

Proposition 2.6 (Shelah [28, p. 99]). Let $\lambda$ be a singular cardinal and let $\kappa=\operatorname{cf}(\lambda)$. Suppose $\operatorname{cov}\left(\lambda, \lambda, \kappa^{+}, \kappa\right)>\lambda^{+}$. Then one can find $y_{\alpha} \in P_{\kappa^{+}}(\lambda)$ for $\alpha<\lambda^{+}$so that for any nonzero $\beta<\lambda^{+}$, there is a one-to-one $h \in$ $\prod_{\alpha<\beta} y_{\alpha}$.

Proof. Select a partition $\left\langle X_{\gamma}: \gamma<\kappa\right\rangle$ of $\lambda$ into $\kappa$ pieces of size $\lambda$. For $\beta \in \lambda^{+}$, let $\beta=\bigcup_{\gamma<\kappa} d_{\gamma}^{\beta}$, where $\left|d_{\gamma}^{\beta}\right|<\lambda$ for all $\gamma<\kappa$. Given $\gamma<\kappa$, define by induction $y_{\alpha}^{\gamma} \in P_{\kappa^{+}}\left(X_{\gamma}\right)$ for $\alpha<\lambda^{+}$so that $y_{\alpha}^{\gamma} \backslash A_{\gamma}^{\beta} \neq \emptyset$ for all $\beta<\lambda^{+}$, where $A_{\gamma}^{\beta}=\bigcup\left\{y_{\zeta}^{\gamma}: \zeta \in \alpha \cap d_{\gamma}^{\beta}\right\}$ (note that $A_{\gamma}^{\beta} \in P_{\lambda}\left(X_{\gamma}\right)$ since 
$\left.\left|A_{\gamma}^{\beta}\right| \leq \kappa \cdot\left|\alpha \cup d_{\gamma}^{\beta}\right|<\lambda\right)$. For $\alpha<\lambda^{+}$, set $y_{\alpha}=\bigcup_{\gamma<\kappa} y_{\alpha}^{\gamma}$. Now suppose $0<\beta<\lambda^{+}$. Define $g: \beta \rightarrow \kappa$ by $g(\alpha)=$ the least $\gamma<\kappa$ such that $\alpha \in d_{\gamma}^{\beta}$. Select

$$
h \in \prod_{\alpha<\beta}\left(y_{\alpha}^{g(\alpha)} \backslash \bigcup\left\{y_{\zeta}^{g(\alpha)}: \zeta \in \alpha \cap d_{g(\alpha)}^{\beta}\right\}\right) .
$$

Then $h$ is easily seen to be one-to-one.

We omit the definition of the function pp, which can be found in $[28$, p. 41].

Proposition 2.7 (Shelah, see e.g. [17] and [12, p. 270]). Let $\lambda$ be a singular cardinal. Then $\operatorname{cov}\left(\lambda, \lambda, \kappa^{+}, 2\right) \geq \operatorname{pp}(\lambda) \geq \lambda^{+}$, where $\kappa=\operatorname{cf}(\lambda)$.

Proposition 2.8. Let $\lambda$ be a singular cardinal, and let $\kappa=\operatorname{cf}(\lambda)$. Then the following hold:

(i) (Shelah $\left[28\right.$, p. 384]) If $\lambda<\omega_{\lambda}$, then $\operatorname{pp}(\lambda)=\operatorname{cov}\left(\lambda, \lambda, \kappa^{+}, 2\right)$.

(ii) (Shelah [29, Claim 1.1]) If $\kappa>\omega$ and $\operatorname{pp}(\lambda)=\lambda^{+}$, then $\operatorname{pp}(\lambda)=$ $\operatorname{cov}\left(\lambda, \lambda, \kappa^{+}, 2\right)$.

(iii) (Shelah [28, p. 369]) Suppose that (a) $\kappa=\omega$, (b) $\operatorname{pp}(\chi)<\lambda$ for every singular cardinal $\chi<\lambda$ with $\operatorname{cf}(\chi)=\omega$, and (c) there is a cardinal $\theta<\lambda$ with the property that $\operatorname{pp}(\pi)=\pi^{+}$for every singular cardinal $\pi$ such that $\theta<\pi<\lambda$ and $\operatorname{cf}(\pi)=\omega_{1}$. Then $\operatorname{pp}(\lambda)=$ $\operatorname{cov}\left(\lambda, \lambda, \kappa^{+}, 2\right)$.

Definition. For two infinite cardinals $\lambda \geq \mu$, we let $u(\mu, \lambda)=$ $\operatorname{cov}(\lambda, \mu, \mu, 2)$.

Proposition 2.9 (Donder-Matet [7]). Let $\lambda, \mu$ and $\kappa$ be three infinite cardinals such that $\lambda \geq \mu=\operatorname{cf}(\mu) \geq \kappa=\operatorname{cf}(\kappa)$. Then $u(\kappa, \lambda) \leq u(\kappa, \mu)$. $u(\mu, \lambda)$.

Note that by Proposition 2.9, if $\lambda$ and $\kappa$ are two infinite cardinals such that $\lambda>\kappa=\operatorname{cf}(\kappa)$, then $u(\kappa, \lambda) \leq u\left(\kappa^{+}, \lambda\right)$.

Proposition 2.10 (folklore). Let $\lambda$ and $\mu$ be two cardinals such that $\lambda \geq \mu=\operatorname{cf}(\mu)$. Then $\lambda^{<\mu}=2^{<\mu} \cdot u(\mu, \lambda)$.

Proposition 2.11 (Shelah [29, Claim 2.1(1)]). Let $\kappa, \rho$ and $\lambda$ be three infinite cardinals such that $\kappa \leq \rho<\lambda$, and let $\theta=\operatorname{cov}\left(\lambda, \rho^{+}, \rho^{+}, \kappa\right)$. Suppose $\theta \geq u(\kappa, \rho)$. Then $u(\kappa, \theta)=u\left(\rho^{+}, \lambda\right)$.

Proposition 2.12. Let $\lambda$ and $\kappa$ be two infinite cardinals such that $\lambda>$ $\kappa=\operatorname{cf}(\kappa)>\operatorname{cf}(\lambda)$. Suppose $u\left(\kappa, \lambda^{+}\right)=\lambda^{+}$. Then $u(\kappa, \lambda)=\lambda^{+}$.

Proof. By Proposition 2.1(vii) and Corollary 2.4,

$$
\lambda<\operatorname{cov}\left(\lambda, \lambda,(\operatorname{cf}(\lambda))^{+}, \operatorname{cf}(\lambda)\right) \leq u(\kappa, \lambda) \leq u\left(\kappa, \lambda^{+}\right)=\lambda^{+} .
$$


Proposition 2.13 (Shelah [30, Conclusion 1.2]). Let $\tau$ be an uncountable strong limit cardinal, and $\lambda>\tau$ be a cardinal. Then there exists an infinite cardinal $\kappa<\tau$ such that $\operatorname{cov}(\lambda, \mu, \mu, \kappa)=\lambda$ for every cardinal $\mu$ with $\kappa<\mu \leq \tau$.

Proposition 2.14 (Gitik-Shelah [11]). Let $2<n<\omega$. Suppose that there are $n$ strong cardinals. Then there exists a generic extension in which (a) $\omega_{\omega}$ is a strong limit cardinal, and (b) setting $\lambda=\omega_{\omega_{n}}$,

$$
u\left(\omega_{i+1}, \lambda\right)=\lambda^{+(i+1)}=\operatorname{cov}\left(\lambda, \omega_{i+1}, \omega_{i+1}, \omega_{i}\right)
$$

for every $i \leq n$.

3. When $\operatorname{cov}(\lambda, \mu, \mu, \kappa)>\lambda^{+}$. In this section we extend the following result of Shelah and Rinot (see the proof of Theorem 3.3 in [26]):

(*) Let $\theta$ be an infinite cardinal such that $u\left(\rho^{+}, \theta\right) \leq \theta^{+}$for every infinite cardinal $\rho<\theta$. Suppose $\mathcal{C} \neq \emptyset$, where $\mathcal{C}$ is the class of all cardinals $\pi>\theta$ such that $u(\mu, \pi)>\pi^{+}$for some successor cardinal $\mu$ with $\omega<\mu<\pi$. Then letting $\nu=\bigcap \mathcal{C}, \nu$ is a singular cardinal. Moreover, $\operatorname{cov}\left(\nu, \nu,(\operatorname{cf}(\nu))^{+}, 2\right)>\nu^{+}$.

Consider the following statement:

(**) Let $\mu$ be a regular uncountable cardinal, and $\theta$ be an infinite cardinal such that $u\left(\rho^{+}, \theta\right) \leq \theta^{++}$for any infinite cardinal $\rho<\theta \cap \mu$. Suppose $\tau>\theta \cdot \mu$ is a cardinal such that $u(\mu, \tau)>\tau^{+}$. Then there is a cardinal $\nu$ such that (a) $\theta<\nu \leq \tau$ and $\operatorname{cf}(\nu)=\omega$, (b) $\operatorname{pp}(\nu)>\nu^{+}$, and (c) $u\left(\rho^{+}, \pi\right) \leq \pi^{+}$for any two cardinals $\pi$ and $\rho$ with $\theta<\pi<\nu$ and $\rho<\pi \cap \mu$.

It is simple to see that $(*)$ follows from $(* *)$ (set $\tau=\bigcap \mathcal{C}$ and $\mu=\rho^{+}$, where $\rho$ is some infinite cardinal with $\rho<\tau$ and $\left.u\left(\rho^{+}, \tau\right)>\tau^{+}\right)$. Now $(* *)$ is concerned with the two-variable function $u(\rho, \psi)(=\operatorname{cov}(\psi, \rho, \rho, \omega))$. Switching to the three-variable function $\operatorname{cov}(\psi, \rho, \rho, \eta)$, we obtain the following, more general result:

Proposition 3.1. Let $\sigma$ be a regular infinite cardinal, $\mu>\sigma$ be a regular cardinal, and $\theta \geq \sigma$ be a cardinal such that $\operatorname{cov}\left(\theta, \rho^{+}, \rho^{+}, \sigma\right) \leq \theta^{++}$for every cardinal $\rho$ with $\sigma \leq \rho<\theta \cap \mu$. Suppose $\tau>\theta$ is a cardinal such that $\operatorname{cov}(\tau, \mu, \mu, \sigma)>\tau^{+}$. Then there is a cardinal $\nu$ such that (a) $\theta<$ $\nu \leq \tau$ and $\operatorname{cf}(\nu)=\sigma$, (b) $\operatorname{pp}(\nu)>\nu^{+}$and $\operatorname{cov}\left(\nu, \nu, \sigma^{+}, \sigma\right)>\nu^{+}$, and (c) $\operatorname{cov}\left(\pi, \rho^{+}, \rho^{+}, \sigma\right) \leq \pi^{+}$for any two cardinals $\pi$ and $\rho$ with $\theta<\pi<\nu$ and $\sigma \leq \rho<\pi \cap \mu$.

We need some preparation. 
Definition. Let $Q$ be a nonempty set of ordinals, and $\left\langle\mu_{i}: i \in Q\right\rangle$ a strictly increasing sequence of infinite cardinals. For $f, g \in \prod_{i \in Q} \mu_{i}$, we let $f<^{*} g$ just in case $|\{i \in Q: f(i) \geq g(i)\}|<|Q|$. Let $\delta$ be an ordinal. By a scale of length $\delta$ on $\prod_{i \in Q} \mu_{i}$ we mean a sequence $\left\langle f_{\alpha}: \alpha<\delta\right\rangle$ of elements of $\prod_{i \in Q} \mu_{i}$ such that (a) $f_{\alpha}<^{*} f_{\beta}$ whenever $\alpha<\beta<\delta$, and (b) for any $g \in \prod_{i \in Q} \mu_{i}$, there is $\alpha<\delta$ such that $g<^{*} f_{\alpha}$.

Lemma 3.2 (Shelah $[28$, p. 55]). Suppose $\nu$ is a singular cardinal of uncountable cofinality $\sigma$, and let $\left\langle\nu_{i}: i\langle\sigma\rangle\right.$ be an increasing continuous sequence of infinite cardinals with supremum $\nu$. Then for some closed unbounded subset $C$ of $\sigma$, there is a scale of length $\nu^{+}$on $\prod_{i \in C} \nu_{i}^{+}$.

The following is essentially due to Liu [17] who proved it for $\kappa=\omega$. We include the proof for completeness.

Proposition 3.3. Let $\kappa$ be a regular infinite cardinal, and $\nu$ be a singular cardinal of cofinality $\sigma>\kappa$. Let $\left\langle\nu_{i}: i\langle\sigma\rangle\right.$ be an increasing continuous sequence of cardinals greater than $\sigma$ with supremum $\nu$. Suppose there is a stationary set $S \subseteq\{i<\sigma: \operatorname{cf}(i) \geq \kappa\}$ such that for each $i \in S$, $\operatorname{cov}\left(\nu_{i}, \nu_{i}, \sigma, \kappa\right) \leq \nu_{i}^{+}$. Then $\operatorname{cov}\left(\nu, \nu, \sigma^{+}, 2\right)=\nu^{+}$.

Proof. By Lemma 3.2 one can find $W \subseteq S$ and $F \subseteq \prod_{i \in W} \nu_{i}^{+}$so that $S \backslash W$ is a nonstationary subset of $\sigma,|F|=\nu^{+}$and for each $g \in \prod_{i \in W} \nu_{i}^{+}$, there is $f \in F$ with $|\{i \in W: g(i) \geq f(i)\}|<\sigma$. For $i \in W$, pick $X_{i} \subseteq P_{\nu_{i}}\left(\nu_{i}\right)$ so that $\left|X_{i}\right| \leq \nu_{i}^{+}$and for any $e \in P_{\sigma}\left(\nu_{i}\right)$, there is $Q \in P_{\kappa}\left(X_{i}\right)$ with $e \subseteq \bigcup Q$. Set $X_{i}=\left\{x_{\alpha}^{i}: \alpha<\nu_{i}^{+}\right\}$. For $a \in P_{\sigma^{+}}(\nu)$, let $a=\left\{\beta_{j}^{a}: j<\sigma\right\}$, and set $a_{i}=\nu_{i} \cap\left\{\beta_{j}^{a}: j<i\right\}$ for each $i \in W$. Now for $f \in F$ and $\xi<\sigma$, let $Z_{f}^{\xi}$ be the set of all $a \in P_{\sigma^{+}}(\nu)$ such that for any $i \in W \backslash \xi$, there is $Q \in P_{\kappa}\left(\left\{x_{\alpha}^{i}: \alpha<f(i)\right\}\right)$ with $a_{i} \subseteq \bigcup Q$.

Claim 1. Let $f \in F$ and $\xi<\sigma$. Then there is $Y_{f}^{\xi} \subseteq P_{\nu}(\nu)$ such that $\left|Y_{f}^{\xi}\right| \leq \sigma$ and $Z_{f}^{\xi} \subseteq \bigcup\left\{P(y): y \in Y_{f}^{\xi}\right\}$.

Proof of Claim 1. For $i \in W \backslash \xi$, let $\left\{x_{\alpha}^{i}: \alpha<f(i)\right\}=\left\{t_{\gamma}^{i}: \gamma<\nu_{i}\right\}$. For $k, l \in \sigma$, set

$$
y_{k}^{l}=\bigcup\left\{t_{\gamma}^{i}: i \in W \backslash \xi, \gamma<\nu_{k} \text { and }\left|t_{\gamma}^{i}\right|<\nu_{l}\right\} .
$$

Put $Y_{f}^{\xi}=\left\{y_{k}^{l}: k, l \in \sigma\right\}$. Now fix $a \in Z_{f}^{\xi}$. For $i \in W \backslash \xi$, select $Q_{i} \in P_{\kappa}\left(\nu_{i}\right)$ so that $a_{i} \subseteq \bigcup_{\gamma \in Q_{i}} t_{\gamma}^{i}$. There must exist $l<\sigma$ and a stationary subset $A$ of $W \backslash \xi$ such that for any $i \in A$ and any $\gamma \in Q_{i},\left|t_{\gamma}^{i}\right|<\nu_{l}$. There must also be $k<\sigma$ and a stationary subset $B$ of $A$ such that $Q_{i} \subseteq \nu_{k}$ for all $i \in B$. Then clearly $a \subseteq y_{k}^{l}$.

Claim 2. $P_{\sigma^{+}}(\nu)=\bigcup\left\{Z_{f}^{\xi}: f \in F\right.$ and $\left.\xi<\sigma\right\}$. 
Proof of Claim 2. Let $a \in P_{\sigma^{+}}(\nu)$. Then for $i \in W$, there is $Q_{i} \in P_{\kappa}\left(X_{i}\right)$ with $a_{i} \subseteq \bigcup Q_{i}$. Select $g \in \prod_{i \in W} \nu_{i}^{+}$so that for any $i \in W, Q_{i} \subseteq\left\{x_{\alpha}^{i}\right.$ : $\alpha \leq g(i)\}$. Then one can find $f \in F$ and $\xi<\sigma$ so that $g(i)<f(i)$ for all $i \in W \backslash \xi$. Clearly $a \in Z_{f}^{\xi}$, which completes the proof of the claim.

Set $A=\bigcup\left\{Y_{f}^{\xi}: f \in F\right.$ and $\left.\xi<\sigma\right\}$. Then clearly, $A \subseteq P_{\nu}(\nu)$ and $|A| \leq|\sigma| \cdot \nu^{+}=\nu^{+}$. Moreover, for each $a \in P_{\sigma^{+}}(\nu)$, by Claim 2 there is $y \in A$ with $a \subseteq y$.

We are now ready to prove our result. For better clarity we distinguish two cases corresponding to Propositions 3.4 and 3.5 below.

Proposition 3.4. Let $\theta$ be an uncountable cardinal such that $u\left(\omega_{1}, \theta\right)$ $\leq \theta^{++}$. Suppose there is a cardinal $\lambda>\theta$ such that $u\left(\omega_{1}, \lambda\right)>\lambda^{+}$, and let $\nu$ be the least such $\lambda$. Then $\operatorname{cf}(\nu)=\omega$ and $\operatorname{pp}(\nu)>\nu^{+}$.

Proof. By Proposition 2.1(viii), $\nu$ is a limit cardinal. It follows from Proposition 2.1(ix) that $\operatorname{cf}(\nu)=\omega$. Moreover, $\operatorname{cov}\left(\nu, \nu, \omega_{1}, 2\right)>\nu^{+}$since by Proposition 2.1(ii),

$$
\operatorname{cov}\left(\nu, \nu, \omega_{1}, 2\right)=\operatorname{cov}\left(\nu, \nu, \omega_{1}, 2\right) \cdot\left(\bigcup_{\theta<\pi<\nu} u\left(\omega_{1}, \pi\right)\right) \geq u\left(\omega_{1}, \nu\right) .
$$

Now suppose to the contrary that $\operatorname{pp}(\nu) \leq \nu^{+}$. Then $\nu=\omega_{\nu}$ by Proposition 2.8(i). Now for every singular cardinal $\chi \leq \theta$ with $\operatorname{cf}(\chi)=\omega$,

$$
\begin{aligned}
\operatorname{pp}(\chi) & \leq \operatorname{cov}\left(\chi, \chi, \omega_{1}, 2\right) \leq \operatorname{cov}\left(\theta, \chi, \omega_{1}, 2\right) \leq \operatorname{cov}\left(\theta, \omega_{1}, \omega_{1}, 2\right)=u\left(\omega_{1}, \theta\right) \\
& \leq \theta^{++}
\end{aligned}
$$

by Propositions 2.1((iii) and (vii)) and 2.7. Moreover, for every cardinal $\pi$ such that $\theta<\pi<\nu$ and $\operatorname{cf}(\pi)=\omega$,

$$
\pi^{+} \leq \operatorname{pp}(\pi) \leq \operatorname{cov}\left(\pi, \pi, \omega_{1}, 2\right) \leq \operatorname{cov}\left(\pi, \omega_{1}, \omega_{1}, 2\right)=u\left(\omega_{1}, \pi\right) \leq \pi^{+} .
$$

by Propositions 2.1((iii) and (vi)) and 2.7. Hence by Propositions 2.7 and $3.3, \operatorname{cov}\left(\eta, \eta, \omega_{2}, 2\right)=\operatorname{pp}(\eta)=\eta^{+}$for any singular cardinal $\eta$ such that $\theta<\eta<\nu$ and $\operatorname{cf}(\eta)=\omega_{1}$. We can now deduce from Proposition 2.8(iii) that $\operatorname{pp}(\nu)=\operatorname{cov}\left(\nu, \nu, \omega_{1}, 2\right)$. Contradiction.

Proposition 3.5. Let $\kappa, \mu, \lambda$ and $\theta$ be four infinite cardinals such that $\operatorname{cf}(\kappa)=\kappa, \kappa \cdot \omega_{1}<\operatorname{cf}(\mu)=\mu \leq \lambda$ and $\kappa \leq \theta<\lambda$. Suppose that (i) $\operatorname{cov}\left(\theta, \rho^{+}, \rho^{+}, \kappa\right) \leq \theta^{++}$for every cardinal $\rho$ with $\kappa \leq \rho<\theta \cap \mu$, and (ii) $\operatorname{cov}(\lambda, \mu, \mu, \kappa)>\lambda^{+}$. Then there exists a cardinal $\nu$ such that (a) $\theta<\nu \leq \lambda$, (b) $\operatorname{cf}(\nu)=\kappa$, (c) $\operatorname{cov}\left(\nu, \nu, \kappa^{+}, \kappa\right)>\nu^{+}$, (d) $\operatorname{cov}\left(\pi, \rho^{+}, \rho^{+}, \kappa\right) \leq \pi^{+}$whenever $\pi$ and $\rho$ are two cardinals such that $\theta<\pi<\nu$ and $\kappa \leq \rho<\pi \cap \mu$, and (e) $\operatorname{pp}(\nu)>\nu^{+}$.

Proof. Let $W$ be the set of all cardinals $\pi>\theta \operatorname{such}$ that $\operatorname{cov}\left(\pi, \rho^{+}, \rho^{+}, \kappa\right)$ $>\pi^{+}$for some cardinal $\rho$ with $\kappa \leq \rho<\pi \cap \mu$. Then $\lambda \in W$ by Propo- 
sition $2.1((\mathrm{vi})$ and $(\mathrm{x}))$. Let $\nu$ be the least element of $W$. Note that by Proposition 2.1((i) and (viii)), $\nu>\theta^{+}$. Let $\sigma$ be the least cardinal $\rho$ such that $\kappa \leq \rho<\nu \cap \mu$ and $\operatorname{cov}\left(\nu, \rho^{+}, \rho^{+}, \kappa\right)>\nu^{+}$. Then $\sigma^{+}<\nu$ by Proposition 2.1(i).

It follows from Proposition 2.1(viii) that $\nu$ is a limit cardinal. Set $\tau=$ $\operatorname{cf}(\nu)$. Let $\left\langle\nu_{i}: i<\tau\right\rangle$ be an increasing continuous sequence of cardinals such that $\theta \cdot \sigma<\nu_{0}$ and $\bigcup_{i<\tau} \nu_{i}=\nu$. Note that $\operatorname{cov}\left(\nu_{i}, \sigma^{+}, \sigma^{+}, \kappa\right) \leq \nu_{i}^{+}$for every $i<\tau$.

Claim 1. $\kappa \leq \tau \leq \sigma$.

Proof of Claim 1. That $\kappa \leq \tau$ follows from Proposition 2.1((vii) and (xi)). For the other inequality use Proposition 2.1((vii) and (ix)).

Claim 2. $\tau=\sigma$.

Proof of Claim 2. Suppose otherwise. Then $\operatorname{cov}\left(\nu, \tau^{+}, \tau^{+}, \kappa\right) \leq \nu^{+}$, so there exists $X \subseteq P_{\tau^{+}}(\nu)$ such that $|X| \leq \nu^{+}$and for every $c \in P_{\tau^{+}}(\nu)$, there is $Q \in P_{\kappa}(X)$ with $c \subseteq \bigcup Q$. For $i<\tau$, select $Y_{i} \subseteq P_{\sigma^{+}}\left(\nu_{i}\right)$ so that $\left|Y_{i}\right| \leq \nu$ and for any $b \in P_{\sigma^{+}}\left(\nu_{i}\right)$, there is $R \in P_{\kappa}\left(Y_{i}\right)$ with $b \subseteq \bigcup R$. Pick an onto function $f_{i}: \nu \rightarrow Y_{i}$. Finally, set $d_{x}=\bigcup_{\delta \in x} \bigcup_{i<\tau} f_{i}(\delta)$ for each $x \in X$. Note that $d_{x} \in P_{\sigma^{+}}(\nu)$. Now given $a \in P_{\sigma^{+}}(\nu)$, select $R_{i} \in P_{\kappa}(\nu)$ for $i<\tau$ so that $a \cap \nu_{i} \subseteq \bigcup_{\delta \in R_{i}} f_{i}(\delta)$. There must be $Q \in P_{\kappa}(X)$ such that $\bigcup_{i<\tau} R_{i} \subseteq \bigcup Q$. Then clearly $a \subseteq \bigcup_{x \in Q} d_{x}$. It follows that $\operatorname{cov}\left(\nu, \sigma^{+}, \sigma^{+}, \kappa\right) \leq|X|$, which yields the desired contradiction.

Claim 3. $\operatorname{cov}\left(\nu, \nu, \sigma^{+}, \kappa\right)>\nu^{+}$.

Proof of Claim 3. Suppose otherwise. Pick $T \subseteq\left\{t \in P_{\nu}(\nu): \theta^{+} \cup \sigma^{+} \subseteq t\right\}$ so that $|T| \leq \nu^{+}$and for any $b \in P_{\sigma^{+}}(\nu)$, there is $H \in P_{\kappa}(T)$ with $b \subseteq \bigcup H$. For $t \in T, \operatorname{cov}\left(|t|, \sigma^{+}, \sigma^{+}, \kappa\right) \leq|t|^{+}$so one can find $Z_{t} \subseteq P_{\sigma^{+}}(t)$ so that $\left|Z_{t}\right| \leq \nu^{+}$and for every $c \in P_{\sigma^{+}}(t)$, there is $G \in P_{\kappa}\left(Z_{t}\right)$ with $c \subseteq \cup G$. Set $Z=\bigcup_{t \in T} Z_{t}$. Then clearly $Z \subseteq P_{\sigma^{+}}(\nu)$. Moreover, $|Z| \leq \nu^{+}$. Now given $b \in P_{\sigma^{+}}(\nu)$, there must be $H \in P_{\kappa}(T)$ with $b \subseteq \bigcup H$. For $t \in T$, select $G_{t} \in P_{\kappa}\left(Z_{t}\right)$ so that $b \cap t \subseteq \bigcup G_{t}$. Then $b \subseteq \bigcup\left(\bigcup_{t \in H} G_{t}\right)$, where $\bigcup_{t \in H} G_{t} \in P_{\kappa}(Z)$. Thus $\operatorname{cov}\left(\nu, \sigma^{+}, \sigma^{+}, \kappa\right) \leq \nu^{+}$, a contradiction.

Claim 4. $\tau=\kappa$.

Proof of Claim 4. Suppose otherwise. Then by Propositions 2.1((iii) and (iv) ) and 3.4, there must be $i<\sigma$ such that $\operatorname{cf}(i)=\kappa$ and $\operatorname{cov}\left(\nu_{i}, \nu_{i}, \sigma, \kappa\right)$ $>\nu_{i}^{+}$. But then by Proposition 2.1((v) and (vi)),

$$
\operatorname{cov}\left(\nu_{i}, \sigma^{+}, \sigma^{+}, \kappa\right) \geq \operatorname{cov}\left(\nu_{i}, \nu_{i}, \sigma^{+}, \kappa\right) \geq \operatorname{cov}\left(\nu_{i}, \nu_{i}, \sigma, \kappa\right)>\nu_{i}^{+} .
$$

Contradiction.

Claim 5. $\operatorname{pp}(\nu)>\nu^{+}$. 
Proof of Claim 5. Suppose otherwise. Then by Propositions 2.1((iii) and (iv)) and 2.7, $\operatorname{cov}\left(\nu, \nu, \kappa^{+}, 2\right)>\operatorname{pp}(\nu)=\nu^{+}$. Hence by Proposition 2.8(ii), $\kappa=\omega$. Now argue exactly as for Proposition 3.4.

Definition. Let $\kappa<\mu$ be two regular infinite cardinals, and $\tau \geq \kappa$ be a cardinal. We let $\theta(\kappa, \tau, \mu)$ denote the least cardinal $\theta \geq \tau$ such that $\operatorname{cov}\left(\theta, \rho^{+}, \rho^{+}, \kappa\right) \leq \theta^{++}$for every cardinal $\rho$ with $\kappa \leq \rho<\theta \cap \mu$.

Clearly $\theta(\kappa, \tau, \mu) \leq \tau<\mu$. Note that if $\kappa=\tau$, then $\theta(\kappa, \tau, \mu)=\tau$. Also note that if $\eta$ is a cardinal such that $\tau \leq \eta \leq \mu$ and $\eta^{<\eta} \leq \eta^{++}$, then $\theta(\kappa, \tau, \mu) \leq \eta$.

Definition. SSH (Shelah's Strong Hypothesis) asserts that $\operatorname{pp}(\lambda)=\lambda^{+}$ for every singular cardinal $\lambda$.

Corollary 3.6 (Shelah ([27] and [28, p. 59]). The following are equivalent:

(i) $\mathrm{SSH}$.

(ii) Given two uncountable cardinals $\lambda$ and $\mu$ such that $\lambda \geq \mu=\operatorname{cf}(\mu)$, $u(\mu, \lambda)$ equals $\lambda$ if $\operatorname{cf}(\lambda) \geq \mu$, and $\lambda^{+}$otherwise.

(iii) For every cardinal $\lambda \geq \omega_{1}$ with $\operatorname{cf}(\lambda)=\omega, u\left(\omega_{1}, \lambda\right) \leq \lambda^{+}$.

Proof. (i) $\Rightarrow$ (ii): By Propositions 2.1, 3.4 and 3.5.

(ii) $\Rightarrow$ (iii): Trivial.

(iii) $\Rightarrow$ (ii): By Propositions 2.1 and 3.5.

(iv) $\Rightarrow$ (i): By Proposition 2.7.

COROLlary 3.7 (Silver [31] for (i) $\Leftrightarrow($ iii)). The following are equivalent:

(i) $\mathrm{SCH}$.

(ii) Given two uncountable cardinals $\lambda$ and $\mu$ such that $\lambda \geq \mu=\operatorname{cf}(\mu)$ and $\lambda>\left(\theta\left(\omega, 2^{\aleph_{0}}, \mu\right)\right)^{+}, u(\mu, \lambda)$ equals $\lambda$ if $\operatorname{cf}(\lambda) \geq \mu$, and $\lambda^{+}$otherwise.

(iii) For every cardinal $\lambda \geq 2^{\aleph_{0}}$ with $\operatorname{cf}(\lambda)=\omega, u\left(\omega_{1}, \lambda\right) \leq \lambda^{+}$.

Proof. (i) $\Rightarrow$ (iii): Trivial.

(iii) $\Rightarrow$ (ii): Assume (iii) holds. Then by Propositions 2.7, 3.4 and 3.5, $u(\mu, \tau) \leq \tau^{+}$whenever $\mu$ and $\tau$ are two cardinals such that $\tau \geq \mu=\operatorname{cf}(\mu)>$ $\omega$ and $\tau>\theta\left(\omega, 2^{\aleph_{0}}, \mu\right)$. That (ii) holds now follows from Proposition 2.1.

(ii) $\Rightarrow(\mathrm{i})$ : It easily follows from (ii) that $u\left((\operatorname{cf}(\lambda))^{+}, \lambda\right) \leq \lambda^{+}$for every infinite cardinal $\lambda$ such that $\lambda>2^{\mathrm{cf}(\lambda)}$.

Proposition 3.8. Suppose SCH holds. Then there is a cardinal $\tau \geq 2^{\aleph_{0}}$ such that for every cardinal $\lambda>\tau^{+}$and every regular uncountable cardinal $\mu \leq \lambda, u(\mu, \lambda)$ equals $\lambda$ if $\operatorname{cf}(\lambda) \geq \mu$, and $\lambda^{+}$otherwise.

Proof. Let $\mathcal{C}$ be the class of all cardinals $\pi \geq 2^{\aleph_{0}}$ such that $u\left(\rho^{+}, \pi\right) \leq$ $\pi^{++}$for every infinite cardinal $\rho<\pi$. Then clearly $\theta\left(\omega, 2^{\aleph_{0}}, \mu\right) \leq \pi$ for every 
$\pi \in \mathcal{C}$ and every regular uncountable cardinal $\mu$. Moreover, for any cardinal $\pi \geq 2^{\aleph_{0}}$ with $\pi \notin \mathcal{C}$, there is an uncountable successor cardinal $\mu \leq \pi$ such that $u(\mu, \lambda)>\lambda^{+}$, where $\lambda=\pi^{++}$. Hence by Corollary 3.7, $\tau$ is as desired if and only if $\tau \in \mathcal{C}$.

To show that $\mathcal{C} \neq \emptyset$, define $\sigma_{n}$ for $n<\omega$ by $\sigma_{0}=2^{\aleph_{0}}$ and $\sigma_{n+1}=$ $\bigcup_{\omega \leq \rho<\sigma_{n}} u\left(\rho^{+}, \sigma_{n}\right)$. Put $\sigma=\bigcup_{n<\omega} \sigma_{n}$. Suppose $\mathcal{C} \cap \sigma=\emptyset$. Then $\sigma_{0}<\sigma_{1}<\cdots$, so $\operatorname{cf}(\sigma)=\omega$. Let $\rho<\sigma$ be an infinite cardinal. Then $\operatorname{cov}\left(\sigma, \rho^{+}, \rho^{+}, \omega_{1}\right)$ $=\sigma$ since $u\left(\rho^{+}, \sigma_{n}\right)<\sigma$ for any $n<\omega$ such that $\rho<\sigma_{n}$, and consequently $u\left(\rho^{+}, \sigma\right) \leq \sigma^{\aleph_{0}}=\sigma^{+}$. Hence $\sigma \in \mathcal{C}$.

4. Covering numbers and normal ideals on $P_{\kappa}(\lambda)$. Throughout this section, $\kappa, \mu$ and $\lambda$ will denote three uncountable cardinals such that $\operatorname{cf}(\kappa)=\kappa \leq \mu<\lambda$.

Let $A_{\kappa, \mu, \lambda}$ assert the existence of $B \in N S_{\kappa, \lambda}^{*}$ such that $N S_{\kappa, \lambda}^{\mu}\left|B=I_{\kappa, \lambda}\right| B$. $A_{\kappa, \mu, \lambda}$ has many interesting consequences. See e.g. [20] where it is shown that $A_{\kappa, \kappa, \lambda}$ implies that $I_{\kappa, \lambda}^{+} \nrightarrow\left(I_{\kappa, \lambda}^{+}, \omega_{1}\right)^{2}$. In this section we show that if $\lambda$ is large enough, then $A_{\kappa, \mu, \lambda}$ holds just in case $\operatorname{cov}\left(\lambda, \mu^{+}, \mu^{+}, \kappa\right)=\lambda$.

Lemma 4.1 (Matet-Péan-Shelah [24]).

$$
\overline{\operatorname{cof}}\left(N S_{\kappa, \lambda}^{\mu}\right)=\overline{\operatorname{cof}}\left(N S_{\kappa, \mu}\right) \cdot \operatorname{cov}\left(\lambda, \mu^{+}, \mu^{+}, \kappa\right) .
$$

Lemma 4.2. Let $A \in N S_{\kappa, \lambda}^{*}$. Then $\overline{\operatorname{cof}}\left(N S_{\kappa, \lambda}^{\mu} \mid A\right) \geq \operatorname{cov}\left(\lambda, \mu^{+}, \mu^{+}, \kappa\right)$.

Proof. Select $t: P_{\omega}(\lambda) \rightarrow P_{\kappa}(\lambda)$ so that $C_{t}^{\kappa, \lambda} \subseteq A$. Fix a family $\mathcal{G}$ of functions from $P_{\omega}(\mu)$ to $P_{\kappa}(\lambda)$ such that $|\mathcal{G}|=\overline{\operatorname{cof}}\left(N S_{\kappa, \lambda}^{\mu} \mid C_{t}^{\kappa, \lambda}\right)$ and for any $h: P_{\omega}(\mu) \rightarrow P_{\kappa}(\lambda)$, there is $X \in P_{\kappa}(\mathcal{G}) \backslash\{\emptyset\}$ with $C_{t}^{\kappa, \lambda} \cap \bigcap_{g \in X} C_{g}^{\kappa, \lambda} \subseteq C_{h}^{\kappa, \lambda}$. For $g \in \mathcal{G}$, set $B_{g}=\mu \cup \bigcup \operatorname{ran}(g)$. Note that $\left|B_{g}\right|=\mu$. For $G \in P_{\omega}(\mathcal{G}) \backslash\{\emptyset\}$, define inductively $B_{G}^{n}$ for $n<\omega$ by $B_{G}^{0}=\bigcup_{g \in G} B_{g}$ and $B_{g}^{n+1}=B_{G}^{n} \cup \bigcup\{t(d)$ : $\left.d \in P_{\omega}\left(B_{G}^{n}\right)\right\}$. Then set $B_{G}=\bigcup_{n<\omega} B_{G}^{n}$. Note that $\left|B_{G}\right|=\mu$. Now fix $D \subseteq \lambda$ with $|D| \leq \mu$. Pick $h: P_{\omega}(\mu) \rightarrow P_{\kappa}(\lambda)$ so that $D \subseteq \bigcup \operatorname{ran}(h)$. There must be $X \in P_{\kappa}(\mathcal{G}) \backslash\{\emptyset\}$ such that $C_{t}^{\kappa, \lambda} \cap \bigcap_{g \in X} C_{g}^{\kappa, \lambda} \subseteq C_{h}^{\kappa, \lambda}$.

For $e \in P_{\omega}(\mu)$, define inductively $s_{n}^{e}$ for $n<\omega$ by $s_{0}^{e}=e \cup \omega$ and

$$
\begin{aligned}
s_{n+1}^{e}= & s_{n}^{e} \cup \bigcup\left\{g(d): g \in X \text { and } d \in P_{\omega}\left(s_{n}^{e} \cap \mu\right)\right\} \\
& \cup \bigcup\left\{t(b): b \in P_{\omega}\left(s_{n}^{e}\right)\right\} .
\end{aligned}
$$

Let us show by induction that $s_{n}^{e} \subseteq \bigcup\left\{B_{G}: G \in P_{\omega}(X) \backslash\{\emptyset\}\right)$. This clearly holds for $n=0$. Now suppose it holds for some $n$. Let $b \in P_{\omega}\left(s_{n}^{e}\right)$. If $b=\emptyset$, then clearly $t(b) \subseteq \bigcup\left\{B_{G}: G \in P_{\omega}(X) \backslash\{\emptyset\}\right.$. Now assume $b \neq \emptyset$. For $\beta \in b$, pick $G_{\beta} \in P_{\omega}(X) \backslash\{\emptyset\}$ so that $\beta \in B_{G_{\beta}}$. Set $Q=\bigcup_{\beta \in b} G_{\beta}$. Note that $Q \in P_{\omega}(X) \backslash\{\emptyset\}$. Given $\beta \in b$, it is easily seen that $B_{G_{\beta}}^{n} \subseteq B_{Q}$ for all $n<\omega$. Hence $b \in P_{\omega}\left(B_{Q}\right)$, and therefore $t(b) \subseteq B_{Q}$. It readily 
follows that $s_{n+1}^{e} \subseteq \bigcup\left\{B_{G}: G \in P_{\omega}(X) \backslash\{\emptyset\}\right\}$. Put $z_{e}=\bigcup_{n<\omega} s_{n}^{e}$. Then $z_{e} \in C_{t}^{\kappa, \lambda} \cap \bigcap_{g \in X} C_{g}^{\kappa, \lambda}$, hence $z_{e} \in C_{h}^{\kappa, \lambda}$. It follows that $h(e) \subseteq z_{e}$, since $e \in P_{\omega}\left(z_{e} \cap \mu\right)$.

Finally,

$$
\begin{aligned}
D & \subseteq \bigcup\left\{h(e): e \in P_{\omega}(\mu)\right\} \subseteq \bigcup\left\{z_{e}: e \in P_{\omega}(\mu)\right\} \\
& \subseteq \bigcup\left\{B_{G}: G \in P_{\omega}(X) \backslash\{\emptyset\}\right\} .
\end{aligned}
$$

Thus $\operatorname{cov}\left(\lambda, \mu^{+}, \mu^{+}, \kappa\right) \leq|\mathcal{G}| \leq \overline{\operatorname{cof}}\left(N S_{\kappa, \lambda}^{\mu} \mid A\right)$.

The following is immediate from the proof of Proposition 3.5 in [24].

LEMma 4.3. Let $\mathcal{K}$ be a family of functions from $P_{\omega}(\lambda)$ to $P_{\kappa}(\lambda)$ with the property that for any $f: P_{\omega}(\mu) \rightarrow P_{\kappa}(\mu)$, one can find $K \in P_{\kappa}(\mathcal{K}) \backslash\{\emptyset\}$ and $h: P_{\omega}(\lambda) \rightarrow P_{\kappa}(\mu)$ with $f \subseteq h$ such that $\bigcap_{k \in K} C_{k}^{\kappa, \lambda} \subseteq C_{h}^{\kappa, \lambda}$. Then $\overline{\operatorname{cof}}\left(N S_{\kappa, \mu}\right) \leq|\mathcal{K}|$.

Lemma 4.4. Let $A \in N S_{\kappa, \lambda}^{*}$. Then $\overline{\operatorname{cof}}\left(N S_{\kappa, \lambda}^{\mu} \mid A\right) \geq \overline{\operatorname{cof}}\left(N S_{\kappa, \mu}\right)$.

Proof. By Lemma 1.1 there is $t: P_{\omega}(\lambda) \rightarrow P_{\kappa}(\lambda)$ such that $C_{t}^{\kappa, \lambda} \subseteq A$. By the same lemma there is a family $\mathcal{Y}$ of functions from $P_{\omega}(\mu)$ to $P_{\kappa}(\lambda)$ such that (a) $|\mathcal{Y}|=\overline{\operatorname{cof}}\left(N S_{\kappa, \lambda}^{\mu} \mid C_{t}^{\kappa, \lambda}\right)$, and (b) for any $B \in\left(N S_{\kappa, \lambda}^{\mu} \mid C_{t}^{\kappa, \lambda}\right)^{*}$, there is $Y \in P_{\kappa}(\mathcal{Y}) \backslash\{\emptyset\}$ with $C_{t}^{\kappa, \lambda} \cap \bigcap_{y \in Y} C_{y}^{\kappa, \lambda} \subseteq B$. For $y \in \mathcal{Y}$, select $\widehat{y}: P_{\omega}(\lambda) \rightarrow P_{\kappa}(\lambda)$ with $y \subseteq \widehat{y}$. Set $\mathcal{K}=\{t\} \cup\{\hat{y}: y \in \mathcal{Y}\}$.

Given $f: P_{\omega}(\mu) \rightarrow P_{\kappa}(\mu)$, define $h: P_{\omega}(\lambda) \rightarrow P_{\kappa}(\mu)$ as follows: $h(e)$ equals $f(e)$ if $e \subseteq \mu$, and $\emptyset$ otherwise. There must be $Y \in P_{\kappa}(\mathcal{Y}) \backslash\{\emptyset\}$ such that $C_{t}^{\kappa, \lambda} \cap \bigcap_{y \in Y} C_{y}^{\kappa, \lambda} \subseteq C_{f}^{\kappa, \lambda}$. We claim that $C_{t}^{\kappa, \lambda} \cap \bigcap_{y \in Y} C_{\widehat{y}}^{\kappa, \lambda} \subseteq C_{h}^{\kappa, \lambda}$. Let $b \in C_{t}^{\kappa, \lambda} \cap \bigcap_{y \in Y} C_{\widehat{y}}^{\kappa, \lambda}$ and $e \in P_{\omega}(b)$. If $e \backslash \mu \neq \emptyset$, then $h(e)=\emptyset \subseteq b$. Otherwise $h(e)=f(e) \subseteq b$, since $b$ belongs to $C_{t}^{\kappa, \lambda} \cap \bigcap_{y \in Y} C_{y}^{\kappa, \lambda}$ and therefore to $C_{f}^{\kappa, \lambda}$. Hence $b \in C_{h}^{\kappa, \lambda}$.

It now follows from Lemma 4.3 that

$$
\overline{\operatorname{cof}}\left(N S_{\kappa, \mu}\right) \leq|\mathcal{K}| \leq \overline{\operatorname{cof}}\left(N S_{\kappa, \lambda}^{\mu} \mid C_{t}^{\kappa, \lambda}\right) \leq \overline{\operatorname{cof}}\left(N S_{\kappa, \lambda}^{\mu} \mid A\right) .
$$

Proposition 4.5. Let $A \in N S_{\kappa, \lambda}^{*}$. Then $\overline{\operatorname{cof}}\left(N S_{\kappa, \lambda}^{\mu} \mid A\right)=\overline{\operatorname{cof}}\left(N S_{\kappa, \lambda}^{\mu}\right)$.

Proof. By Lemmas 4.1, 4.2 and 4.4.

Lemma 4.6 (Matet-Péan-Shelah [24]). If $\overline{\operatorname{cof}}\left(N S_{\kappa, \lambda}^{\mu}\right) \leq \lambda$, then $N S_{\kappa, \lambda}^{\mu} \mid A$ $=I_{\kappa, \lambda} \mid A$ for some $A \in N S_{\kappa, \lambda}^{*}$.

Proposition 4.7. The following are equivalent:

(i) $\overline{\operatorname{cof}}\left(N S_{\kappa, \mu}\right) \leq \lambda=\operatorname{cov}\left(\lambda, \mu^{+}, \mu^{+}, \kappa\right)$.

(ii) There is $A \in N S_{\kappa, \lambda}^{*}$ such that $N S_{\kappa, \lambda}^{\mu}\left|A=I_{\kappa, \lambda}\right| A$. 
Proof. (i) $\Rightarrow$ (ii): By Lemmas 4.1 and 4.6.

(ii) $\Rightarrow(\mathrm{i})$ : By Lemma 4.1 and Propositions 2.1((i) and (ii)) and 4.5.

Note that if $\kappa \leq \operatorname{cf}(\lambda)<\lambda$, then $\operatorname{cov}\left(\lambda,(\operatorname{cf}(\lambda))^{+},(\operatorname{cf}(\lambda))^{+}, \kappa\right) \neq \lambda$ by Propositions 2.1 and 2.2, and therefore by Proposition 4.7, $N S_{\kappa, \lambda}^{\operatorname{cf}(\lambda)} \mid A \neq$ $I_{\kappa, \lambda} \mid A$ for every $A \in N S_{\kappa, \lambda}^{*}$.

There is a version of Proposition 4.7 for $N S S_{\kappa, \lambda}$.

Proposition 4.8. Suppose $\lambda$ is a regular limit cardinal. Then the following are equivalent:

(i) $\overline{\operatorname{cof}}\left(N S_{\kappa, \nu}\right) \leq \lambda=\operatorname{cov}\left(\lambda, \nu^{+}, \nu^{+}, \kappa\right)$ for every cardinal $\nu$ with $\kappa \leq$ $\nu<\lambda$.

(ii) There is $C \in N S_{\kappa, \lambda}^{*}$ such that $N S S_{\kappa, \lambda}\left|C=I_{\kappa, \lambda}\right| C$.

Proof. (i) $\Rightarrow$ (ii): Let $Z$ be the set of all cardinals $\nu$ with $\kappa \leq \nu<\lambda$. Clearly, $N S S_{\kappa, \lambda}=\bigcup_{\nu \in Z} N S_{\kappa, \lambda}^{\nu}$. For each $\nu \in Z$, by Proposition 4.7 there is $A_{\nu} \in N S_{\kappa, \lambda}^{*}$ such that $N S_{\kappa, \lambda}^{\nu}\left|A_{\nu}=I_{\kappa, \lambda}\right| A_{\nu}$. Then

$$
C=\left\{a \in P_{\kappa}(\lambda): \forall \nu \in a \cap Z\left(a \in A_{\nu}\right)\right\}
$$

is as desired.

(ii) $\Rightarrow$ (i): By Proposition 4.7.

Lemma 4.9 (Matet-Péan-Shelah [23]). Suppose $\kappa \leq \delta<\lambda$. Then $N S_{\kappa, \lambda}^{\delta}=N S_{\kappa, \lambda}^{|\delta|} \mid D$ for some $D \in\left(N S_{\kappa, \lambda}^{\delta}\right)^{*}$.

Proposition 4.10. Suppose $\lambda$ is a successor cardinal, say $\lambda=\nu^{+}$. Then the following are equivalent:

(i) $\overline{\operatorname{cof}}\left(N S_{\kappa, \nu}\right) \leq \lambda$.

(ii) There is $C \in N S_{\kappa, \lambda}^{*}$ such that $N S S_{\kappa, \lambda}\left|C=I_{\kappa, \lambda}\right| C$.

Proof. (i) $\Rightarrow$ (ii): Let $Z$ be the set of all ordinals $\delta$ with $\nu \leq \delta<\lambda$. Note that $N S S_{\kappa, \lambda}=\bigcup_{\delta \in Z} N S_{\kappa, \lambda}^{\delta}$. Since by Proposition 2.1, $\operatorname{cov}\left(\lambda, \nu^{+}, \nu^{+}, \kappa\right)=\lambda$, Proposition 4.7 yields $A \in N S_{\kappa, \lambda}^{*}$ such that $N S_{\kappa, \lambda}^{\nu}\left|A=I_{\kappa, \lambda}\right| A$. For each $\delta \in Z$, by Lemma 4.9 there is $D_{\delta} \in N S_{\kappa, \lambda}^{*}$ such that $N S_{\kappa, \lambda}^{\delta}=N S_{\kappa, \lambda}^{\nu} \mid D_{\delta}$. Then $C=\left\{a \in A: \forall \delta \in a \cap Z\left(a \in D_{\delta}\right)\right\}$ is as desired.

(ii) $\Rightarrow($ i): By Proposition 4.7.

5. Various notions of compactness. In this section we review several notions of compactness and consider their impact on cardinal arithmetic. We start with mild ineffability.

Definition. Let $\kappa$ and $\lambda$ be two cardinals such that $\omega \leq \operatorname{cf}(\kappa)=\kappa \leq \lambda$. $\kappa$ is mildly $\lambda$-ineffable if given $t_{a}: a \rightarrow 2$ for $a \in P_{\kappa}(\lambda)$, there is $g: \lambda \rightarrow 2$ with the property that for any $b \in P_{\kappa}(\lambda)$, there is $a \in P_{\kappa}(\lambda)$ such that $b \subseteq a$ and $t_{a} \uparrow b=g\lceil b$. 
Proposition 5.1 (Carr [4]). Let $\kappa$ and $\lambda$ be two infinite cardinals such that $\mathrm{cf}(\kappa)=\kappa \leq \lambda$. Then the following hold:

(i) Suppose $\kappa$ is mildly $\lambda$-ineffable. Then $\kappa$ is mildly $\lambda^{\prime}$-ineffable for every cardinal $\lambda^{\prime}$ with $\kappa \leq \lambda^{\prime}<\lambda$.

(ii) For $\lambda=\kappa, \kappa$ is mildly $\lambda$-ineffable if and only if it is weakly compact.

The following is essentially due to Di Prisco and Zwicker [6].

Lemma 5.2. Let $\kappa$ and $\lambda$ be two infinite cardinals such that $\operatorname{cf}(\kappa)=\kappa$ $\leq \lambda$. Then the following are equivalent:

(i) $\kappa$ is mildly $\lambda$-ineffable.

(ii) Let $\left\langle Q_{\alpha}: \alpha<\lambda\right\rangle$ be a sequence of partitions of $P_{\kappa}(\lambda)$ into fewer than $\kappa$ pieces. Then there is $h \in \prod_{\alpha<\lambda} Q_{\alpha}$ such that $\bigcap_{\alpha \in e} h(\alpha) \in I_{\kappa, \lambda}^{+}$for every $e \in P_{\kappa}(\lambda) \backslash\{\emptyset\}$.

Next we consider the uniform filter property $\mathrm{UF}_{\pi}$.

DeFinition. Let $\pi$ be a regular infinite cardinal. We say that $\mathrm{UF}_{\pi}$ holds if for any sequence $\left\langle Q_{i}: i<\pi\right\rangle$ of partitions of $\pi$ into countably many pieces, there is $h \in \prod_{i<\pi} Q_{i}$ such that $\left|\bigcap_{i \in e} h(i)\right|=\pi$ for any $e \in P_{\omega}(\pi) \backslash\{\emptyset\}$.

Proposition 5.3. Let $\kappa \leq \pi$ be two regular uncountable cardinals such that $\kappa$ is mildly $\pi$-ineffable. Then $\mathrm{UF}_{\pi}$ holds.

Proof. By Lemma 5.2.

Let us now consider the transversal property $\operatorname{PT}\left(\pi, \omega_{1}\right)$.

Definition. For a regular infinite cardinal $\pi, \operatorname{PT}\left(\pi, \omega_{1}\right)$ means that for any size $\pi$ family of countable sets without a transversal (i.e. a one-toone choice function), there exists a subfamily of size less than $\pi$ without a transversal.

The following is readily checked.

Proposition 5.4. Let $\pi$ be a regular infinite cardinal such that $\mathrm{UF}_{\pi}$ holds. Then $\mathrm{PT}\left(\pi, \omega_{1}\right)$ holds.

Proposition 5.5 (Magidor-Shelah [18]).

(i) Let $\pi<\omega_{\omega^{2}+1}$ be a regular infinite cardinal. Then $\mathrm{PT}\left(\pi, \omega_{1}\right)$ does not hold.

(iii) It is consistent (relative to infinitely many supercompact cardinals) that $\mathrm{PT}\left(\omega_{\omega^{2}+1}, \omega_{1}\right)$ holds.

(iii) Let $\pi$ be a regular infinite cardinal such that $\mathrm{PT}\left(\pi, \omega_{1}\right)$ does not hold. Then $\mathrm{PT}\left(\omega_{\pi+1}, \omega_{1}\right)$ does not hold.

(iv) It is consistent (relative to infinitely many supercompact cardinals) that $\operatorname{PT}\left(\pi, \omega_{1}\right)$ holds for every regular infinite cardinal $\pi$ greater 
than the first cardinal fixed point (i.e. the least cardinal $\kappa$ such that $\left.\kappa=\omega_{\kappa}\right)$.

Proposition 5.6 (Shelah [28, p. 99]). Let $\lambda$ be a singular cardinal of cofinality $\omega$ such that $\operatorname{PT}\left(\lambda^{+}, \omega_{1}\right)$ holds. Then $\operatorname{cov}\left(\lambda, \lambda, \omega_{1}, 2\right)=\lambda^{+}$.

Proof. By Proposition 2.6.

COROllary 5.7. Let $\kappa$ be a regular uncountable cardinal such that $u\left(\rho^{+}, \kappa\right) \leq \kappa^{++}$for every infinite cardinal $\rho<\kappa$. Further, let $\lambda>\kappa^{+}$be a cardinal such that $\mathrm{PT}\left(\nu^{+}, \omega_{1}\right)$ holds for every cardinal $\nu$ such that $\kappa<\nu<\lambda$ and $\operatorname{cf}(\nu)=\omega$. Finally, let $\mu \leq \lambda$ be a regular uncountable cardinal. Then the following hold:

(i) Suppose $\operatorname{cf}(\lambda)>\omega$. Then $u(\mu, \lambda)$ equals $\lambda$ if $\operatorname{cf}(\lambda) \geq \mu$, and $\lambda^{+}$ otherwise.

(ii) Suppose $\operatorname{cf}(\lambda)=\omega$. Then $\operatorname{cov}\left(\lambda, \mu, \mu, \omega_{1}\right)=\lambda$ and $u(\mu, \lambda)=u\left(\omega_{1}, \lambda\right)$.

Proof. (i) By Proposition 2.1 and Corollary 2.4 it suffices to show that $u(\mu, \sigma) \leq \sigma^{+}$for every cardinal $\sigma$ with $\mu \cdot \kappa^{+} \leq \sigma \leq \lambda$. For $\mu \geq \kappa$, this readily follows from Propositions 2.7, 3.4, 3.5 and 5.6. Now suppose $\mu<\kappa<\sigma \leq \lambda$. Then by Propositions 2.1 and 2.9,

$$
u(\mu, \sigma) \leq u(\mu, \kappa) \cdot u(\kappa, \sigma) \leq \kappa^{++} \cdot \sigma^{+}=\sigma^{+} .
$$

(ii) We know from (i) that $u(\tau, \sigma) \leq \sigma^{+}$for every regular uncountable cardinal $\tau<\lambda$ and every cardinal $\sigma$ with $\tau \cdot \kappa^{+} \leq \sigma<\lambda$. Hence by Proposition 2.1,

$$
\operatorname{cov}\left(\lambda, \mu, \mu, \omega_{1}\right)=\bigcup_{\mu \cdot \kappa^{+} \leq \sigma<\lambda} \operatorname{cov}\left(\sigma, \mu, \mu, \omega_{1}\right)=\lambda .
$$

It follows that

$$
u(\mu, \lambda) \leq u\left(\omega_{1}, \operatorname{cov}\left(\lambda, \mu, \mu, \omega_{1}\right)\right)=u\left(\omega_{1}, \lambda\right) .
$$

The proof is concluded by appealing to Proposition 2.9. In case $\mu<\kappa$, we get

$$
u\left(\omega_{1}, \lambda\right) \leq u\left(\omega_{1}, \mu\right) \cdot u(\mu, \lambda) \leq u\left(\omega_{1}, \kappa\right) \cdot u(\mu, \lambda) \leq \kappa^{++} \cdot u(\mu, \lambda)=u(\mu, \lambda) .
$$

Otherwise,

$$
u\left(\omega_{1}, \lambda\right) \leq u\left(\omega_{1}, \kappa\right) \cdot u(\kappa, \mu) \cdot u(\mu, \lambda) \leq \kappa^{++} \cdot \mu^{+} \cdot u(\mu, \lambda)=u(\mu, \lambda) .
$$

Finally, we consider the weak Rado conjecture $\mathrm{w} \mathrm{RC}(\sigma, \pi)$.

Definition. Given two uncountable cardinals $\sigma \leq \pi, \operatorname{wRC}(\sigma, \pi)$ asserts the following. Let $(T,<)$ be a tree of size $\pi$ with the property that any subtree of size less than $\sigma$ is special (i.e. is the union of countably many antichains). Then $T$ is not Baire (i.e. there is a sequence $\left\langle D_{i}: i<\omega\right\rangle$ of cofinal subsets of $(T,<)$ such that (a) for any $i<\omega$ and any $t \in D_{i}$, $\left\{t^{\prime} \in T: t<t^{\prime}\right\} \subseteq D_{i}$, and (b) $\left.\bigcap_{i<\omega} D_{i}=\emptyset\right)$. 
The following is easily verified.

PROPOSITION 5.8. Let $\pi$ be a regular uncountable cardinal such that $\mathrm{UF}_{\pi}$ holds. Then $\operatorname{wRC}(\pi, \pi)$ holds (in fact, any nonspecial tree of size $\pi$ has a nonspecial subtree of size less than $\pi$ ).

Proposition 5.9.

(i) (Todorcevic [35]) Suppose $\mathrm{wRC}\left(\lambda^{+}, \lambda^{\aleph_{0}}\right)$ holds, where $\lambda$ is a singular cardinal of cofinality $\omega$. Then $\lambda^{\aleph_{0}}=\lambda^{+}$.

(ii) (Todorcevic [34]) It is consistent (relative to a supercompact cardinal) that $\mathrm{wRC}\left(\omega_{2}, \pi\right)$ holds for every cardinal $\pi \geq \omega_{2}$.

(iii) (Todorcevic [35]) Suppose $\mathrm{wRC}\left(\omega_{2}, \pi\right)$ holds for every cardinal $\pi \geq \omega_{2}$. Then $2^{\aleph_{0}} \leq \aleph_{2}$.

6. Weakly $\lambda^{+}$-saturated ideals on $P_{\kappa}(\lambda)$. Throughout this section $\kappa$ and $\lambda$ will denote two uncountable cardinals such that $\operatorname{cf}(\kappa)=\kappa \leq \lambda$.

Lemma 6.1 (folklore). For every cardinal $\tau$, the following hold:

(i) There is a (normal) $\tau$-saturated ideal on $P_{\kappa}(\kappa)$ if and only if there is one on $\kappa$.

(ii) Let $\sigma$ be a cardinal with $\kappa \leq \sigma<\lambda$. If there is a ( $\sigma$-normal) (weakly) $\tau$-saturated ideal on $P_{\kappa}(\lambda)$, then there is a (normal) (weakly) $\tau$ saturated ideal on $P_{\kappa}(\sigma)$.

Proof. (i) Define $f: \kappa \rightarrow P_{\kappa}(\kappa)$ and $g: P_{\kappa}(\kappa) \rightarrow \kappa$ by $f(\alpha)=\alpha$ and $g(a)=\bigcup a$. If $J$ is a (normal) $\tau$-saturated on $\kappa$ (respectively, on $P_{\kappa}(\kappa)$ ), then $f(J)$ (respectively, $g(J))$ is a (normal) $\tau$-saturated ideal on $P_{\kappa}(\kappa)$ (respectively, on $\kappa$ ).

(ii) Define $p: P_{\kappa}(\lambda) \rightarrow P_{\kappa}(\sigma)$ by $p(a)=a \cap \sigma$. If $J$ is a ( $\sigma$-normal) (weakly) $\tau$-saturated ideal on $P_{\kappa}(\lambda)$, then $p(J)$ is a (normal) (weakly) $\tau$ saturated ideal on $P_{\kappa}(\sigma)$.

LEMMA 6.2 .

(i) (Abe [2]) Suppose that $\operatorname{cf}(\lambda)=\lambda$ and there is a weakly normal ideal on $P_{\kappa}(\lambda)$. Then $u(\kappa, \lambda)=\lambda$.

(ii) (Usuba [38]) Suppose that $\operatorname{cf}(\lambda)=\lambda$ and there is a weakly $\lambda$-saturated ideal on $P_{\kappa}(\lambda)$. Then there is a weakly normal ideal on $P_{\kappa}(\lambda)$.

Proposition 6.3 (Usuba [38]). Suppose that $\operatorname{cf}(\lambda) \geq \kappa$ and there is a weakly $\lambda$-saturated ideal on $P_{\kappa}(\lambda)$. Then $u(\kappa, \lambda)=\lambda$.

Proof. In case $\lambda$ is regular the result is immediate from Lemma 6.2. Now assume $\lambda$ is singular. By Lemmas 1.4(iii) and 6.1(ii) we may find a cardinal $\tau<\lambda$ such that for any cardinal $\sigma$ with $\kappa \leq \sigma<\lambda$, there exists a weakly $\tau$-saturated ideal on $P_{\kappa}(\sigma)$. Hence by Lemma $6.2, u(\kappa, \nu)=\nu$ for every 
regular cardinal $\nu$ with $\kappa \cdot \tau \leq \nu<\lambda$. The desired conclusion now follows from Proposition 2.1.

Definition. For a cardinal $\chi, \mathcal{A}_{\kappa, \lambda}^{\chi}$ asserts the existence of a size $\chi$ subset $A$ of $P_{\kappa}(\lambda)$ such that $|A \cap P(a)|<\kappa$ for every $a \in P_{\kappa}(\lambda)$.

Lemma 6.4. Let $\chi>\lambda$ be a cardinal such that $\mathcal{A}_{\kappa, \lambda}^{\chi}$ holds. Then there is $f: P_{\kappa}(\lambda) \rightarrow P_{\kappa}(\chi)$ such that (a) $f$ is an isomorphism from $\left(P_{\kappa}(\lambda), \subset\right)$ onto $(\operatorname{ran}(f), \subset)$, (b) $\lambda \cap f(a)=a$ for every $a \in P_{\kappa}(\lambda)$, and (c) $f^{-1}(X) \in I_{\kappa, \lambda}$ for every $X \in I_{\kappa, \chi}$.

Proof. Pick $z_{\alpha} \in P_{\kappa}(\lambda)$ for $\lambda \leq \alpha<\chi$ so that (i) $z_{\alpha^{\prime}} \neq z_{\alpha}$ for all $\alpha^{\prime} \neq \alpha$, and (ii) $\left|\left\{\alpha: z_{\alpha} \subseteq a\right\}\right|<\kappa$ for every $a \in P_{\kappa}(\lambda)$. Now define $f: P_{\kappa}(\lambda) \rightarrow P_{\kappa}(\chi)$ by $f(a)=a \cup x_{a}$, where $x_{a}=\left\{\alpha: z_{\alpha} \subseteq a\right\}$ if $\kappa$ is a successor cardinal, and $x_{a}=\left\{\alpha: z_{\alpha} \in P_{|a \cap \kappa|}(a)\right\}$ otherwise.

The following is immediate from Lemma 6.4.

Proposition 6.5. Let $\chi>\lambda$ be a cardinal such that $\mathcal{A}_{\kappa, \lambda}^{\chi}$ holds, and $\sigma$ be a cardinal. If there is a (normal) (weakly) $\sigma$-saturated ideal on $P_{\kappa}(\lambda)$, then there is a ( $\lambda$-normal) (weakly) $\sigma$-saturated ideal on $P_{\kappa}(\chi)$.

Let us remark this in passing:

Proposition 6.6. Let $J$ be a seminormal, weakly $\lambda^{+}$-saturated ideal on $P_{\kappa}\left(\lambda^{+}\right)$. Then $J$ is $\lambda^{+}$-saturated.

Proof. Suppose otherwise. Select $A_{\alpha} \in J^{+}$for $\alpha<\lambda^{+}$so that $A_{\beta} \cap A_{\alpha} \in$ $J$ for all $\beta<\alpha$. For $\alpha<\lambda^{+}$, pick a bijection $i_{\alpha}: \alpha \rightarrow|\alpha|$, and set

$$
\begin{aligned}
& E_{\alpha}=\left\{a \in P_{\kappa}\left(\lambda^{+}\right): \forall \beta \in a \cap \alpha\left(i_{\alpha}(\beta) \in a\right)\right\}, \\
& X_{\alpha}=\left\{a \in P_{\kappa}\left(\lambda^{+}\right): \exists \gamma \in a \cap|\alpha|\left(a \in A_{i_{\alpha}^{-1}(\gamma)} \cap A_{\alpha}\right)\right\}, \\
& B_{\alpha}=\left\{a \in\left(A_{\alpha} \cap E_{\alpha}\right) \backslash X_{\alpha}: \alpha \in a\right\} .
\end{aligned}
$$

Then clearly $B_{\alpha} \in J^{+}$for all $\alpha<\lambda^{+}$. Moreover, $B_{\beta} \cap B_{\alpha}=\emptyset$ whenever $\beta<\alpha<\lambda^{+}$. This is a contradiction.

Lemma 6.7 (Matet [22]). Suppose $\kappa$ is a limit cardinal and $\operatorname{cf}(\lambda)<\kappa$. Then $\mathcal{A}_{\kappa, \lambda}^{\lambda^{+}}$holds.

Proposition 6.8. Suppose that $\kappa$ is a limit cardinal, $\operatorname{cf}(\lambda)<\kappa$ and there is a weakly $\lambda^{+}$-saturated ideal on $P_{\kappa}(\lambda)$. Then $u(\kappa, \lambda)=\lambda^{+}$.

Proof. By Propositions 6.3 and 6.5 and Lemma 6.7.

Let us now consider the case when $\kappa$ is a successor cardinal.

Lemma 6.9 (Matsubara [25]). Suppose $\kappa$ is a successor cardinal. Then no ideal on $P_{\kappa}(\lambda)$ is weakly $\lambda$-saturated.

Note that the result is optimal if $\lambda^{<\kappa}=\lambda$. 
Proposition 6.10. Suppose $\kappa$ is a successor cardinal. Let $\chi>\lambda$ be a cardinal such that $\mathcal{A}_{\kappa, \lambda}^{\chi}$ holds. Then no ideal on $P_{\kappa}(\lambda)$ is weakly $\chi$-saturated.

Proof. By Proposition 6.5 and Lemma 6.9.

Lemma 6.11 (Shelah, see [24]). Suppose that $\operatorname{cf}(\lambda)<\kappa$, and $\chi>\lambda$ is a cardinal such that $u\left(\lambda^{+}, \chi\right)<\operatorname{cov}(\lambda, \lambda, \kappa, 2)$. Then $\mathcal{A}_{\kappa, \lambda}^{\chi}$ holds.

Proposition 6.12. Suppose that $\kappa$ is a successor cardinal, $\operatorname{cf}(\lambda)<\kappa$, and $\chi>\lambda$ is a cardinal such that there exists a weakly $\chi$-saturated ideal on $P_{\kappa}(\lambda)$. Then $u\left(\lambda^{+}, \chi\right) \geq \operatorname{cov}(\lambda, \lambda, \kappa, 2)$.

Proof. By Proposition 6.10 and Lemma 6.11.

In particular, if $\kappa$ is a successor cardinal, $\operatorname{cf}(\lambda)<\kappa$ and there exists a weakly $\lambda^{+}$-saturated ideal on $P_{\kappa}(\lambda)$, then $\operatorname{cov}(\lambda, \lambda, \kappa, 2)=\lambda^{+}$. For more concerning the case when $\kappa$ is a successor cardinal, see [22] and [38].

The following extends Abe's result [2] that the existence of a $\kappa^{+}$-saturated ideal on $P_{\kappa}(\lambda)$ implies that SCH holds between $2^{<\kappa}$ and $\lambda$.

Proposition 6.13. Let $\tau$ and $\mu$ be two cardinals such that $\tau<\lambda$ and $\omega<\mu=\operatorname{cf}(\mu)$. Suppose that $(\theta(\omega, \kappa \cdot \tau, \mu))^{+}<\lambda$ and there exists a $\tau$ saturated ideal on $P_{\kappa}(\lambda)$. Then $u(\mu, \lambda)$ equals $\lambda$ if $\operatorname{cf}(\lambda) \geq \mu$, and $\lambda^{+}$otherwise.

Proof. By Lemma 6.1 and Proposition 6.8, $\operatorname{cov}\left(\nu, \nu, \omega_{1}, \omega\right) \leq \nu^{+}$for any cardinal $\nu$ such that $\theta(\omega, \kappa \cdot \tau, \mu)<\nu \leq \lambda$ and $\operatorname{cf}(\nu)=\omega$. Hence by Propositions 3.4 and 3.5, $u(\mu, \sigma) \leq \sigma^{+}$for each cardinal $\sigma$ with $\mu \cup \theta(\omega, \kappa \cdot \tau, \mu)<$ $\sigma \leq \lambda$. The desired conclusion easily follows.

7. $<\kappa$-saturated ideals on $P_{\kappa}(\lambda)$. Throughout this section, $\kappa$ and $\lambda$ will denote two uncountable cardinals such that $\mathrm{cf}(\kappa)=\kappa \leq \lambda$.

Using a result of Shelah we will prove that if there is a $\tau$-saturated ideal on $P_{\kappa}(\lambda)$ for some $\tau<\kappa$, then SSH holds between $\kappa$ and $\lambda$.

Lemma 7.1 (Shelah [29]). Let $\tau<\kappa$ be a regular infinite cardinal. Suppose there exists a $\tau$-saturated ideal on $\kappa$. Then $u(\tau, \sigma)<\kappa$ for every cardinal $\sigma$ with $\tau \leq \sigma<\kappa$.

Lemma 7.2. Let $\tau$ and $\mu$ be two regular cardinals such that $\omega \leq \tau<\kappa \leq$ $\mu \leq \lambda$. Suppose there exists a $\tau$-saturated ideal on $P_{\kappa}(\lambda)$. Then $\operatorname{cov}(\lambda, \mu, \mu, \tau)$ $\leq \lambda^{+}$.

Proof. By Proposition 2.1 and Lemma 7.1, $u\left(\rho^{+}, \kappa\right)=\kappa$ for every cardinal $\rho$ with $\tau \leq \rho<\kappa$. Moreover, by Lemmas 1.4 and 6.1 and Proposition 6.8, $\operatorname{cov}\left(\nu, \nu, \tau^{+}, \tau\right) \leq u(\kappa, \nu)=\nu^{+}$for every cardinal $\nu$ such that $\kappa<\nu \leq \lambda$ and $\operatorname{cf}(\nu)=\tau$. The desired conclusion is now immediate from Proposition 3.5. 
Proposition 7.3. Suppose $P_{\kappa}(\lambda)$ carries a $\tau$-saturated ideal for some cardinal $\tau<\kappa$. Then for every regular cardinal $\mu$ such that $\kappa<\mu \leq \lambda$, $u(\mu, \lambda)$ equals $\lambda$ if $\operatorname{cf}(\lambda) \geq \mu$, and $\lambda^{+}$otherwise.

Proof. By Proposition 2.1 it suffices to show that $u\left(\chi^{+}, \sigma\right) \leq \sigma^{+}$whenever $\chi$ and $\sigma$ are two cardinals such that $\kappa \leq \chi<\sigma \leq \lambda$. Now given such $\chi$ and $\sigma$,

$$
u(\kappa, \chi) \leq \chi^{+} \leq \sigma \leq \operatorname{cov}\left(\sigma, \chi^{+}, \chi^{+}, \kappa\right) \leq \sigma^{+}
$$

by Proposition 6.3 and Lemma 7.2. Hence by Propositions 2.1, 2.11, 6.3 and 6.8 ,

$$
u\left(\chi^{+}, \sigma\right) \leq u\left(\kappa, \sigma^{+}\right)=\sigma^{+} \cdot u(\kappa, \sigma)=\sigma^{+} .
$$

Next we consider some cases when $\mathcal{A}_{\kappa, \lambda}^{\chi}$ holds.

LEMMA 7.4 (Solovay [32]).

(i) Suppose that for some cardinal $\tau \leq \kappa^{+}, \kappa$ carries a $\tau$-saturated ideal. Then $\kappa$ carries a normal $\tau$-saturated ideal.

(ii) Let $\tau<\kappa$ be a regular uncountable cardinal, and $H$ be a normal $\tau$-saturated ideal on $\kappa$. Then for every cardinal $\nu<\kappa$ and every $F: \kappa \times \kappa \rightarrow \nu$, there is $A \in H^{*}$ such that

$$
\mid\{F(\alpha, \beta): \alpha, \beta \in A \text { and } \alpha<\beta\} \mid<\tau .
$$

Proposition 7.5. Let $\nu$ and $\chi$ be two cardinals such that $\omega \leq \nu=$ $\operatorname{cf}(\nu)<\kappa<\lambda<\chi$. Suppose that $\kappa \rightarrow[\kappa]_{\nu,<\nu}^{2}$ and there is $f_{\alpha}: \nu \rightarrow \lambda$ for $\alpha<\chi$ such that $\left|\left\{i<\nu: f_{\alpha}(i)=f_{\beta}(i)\right\}\right|<\tau$ whenever $\alpha<\beta<\chi$. Then $\mathcal{A}_{\kappa, \lambda}^{\chi}$ holds.

Proof. Select a bijection $t: \nu \times \lambda \rightarrow \lambda$. For $\alpha<\chi$, set $a_{\alpha}=\left\{t\left(i, f_{\alpha}(i)\right)\right.$ : $i<\nu\}$. Now fix $e \subseteq \chi$ with o.t. $(e)=\kappa$. Define $F:\{(\alpha, \beta) \in e \times e: \alpha<\beta\} \rightarrow$ $\nu$ by $F(\alpha, \beta)=$ the least $j<\nu$ such that $f_{\alpha}(i) \neq f_{\beta}(i)$ whenever $j \leq i<\nu$. There must be $k<\nu$ and $d \subseteq e$ such that $|d|=\kappa$ and $F(\alpha, \beta) \leq k$ for every $(\alpha, \beta) \in d \times d$ with $\alpha<\beta$. For $\alpha \in d$, put $b_{\alpha}=\left\{t\left(i, f_{\alpha}(i)\right): k \leq i<\nu\right\}$. Then clearly $b_{\alpha} \cap b_{\beta}=\emptyset$ for any $(\alpha, \beta) \in d \times d$ with $\alpha<\beta$. Hence $\left|\bigcup_{\alpha \in e} a_{\alpha}\right|=\kappa$.

Definition. $\operatorname{ADS}_{\lambda}$ asserts the existence of a sequence $\left\langle a_{\alpha}: \alpha<\lambda^{+}\right\rangle$ such that (a) $a_{\alpha}$ is a cofinal subset of $\lambda$ of order type $\operatorname{cf}(\lambda)$, and (b) for any $\beta<\lambda^{+}$, there is $g_{\beta}: \beta \rightarrow \lambda$ such that

$$
\left(a_{\alpha} \backslash g_{\beta}(\alpha)\right) \cap\left(a_{\gamma} \backslash g_{\beta}(\gamma)\right)=\emptyset
$$

whenever $\alpha<\gamma<\beta$.

Assuming $\operatorname{cf}(\lambda)<\kappa, \operatorname{ADS}_{\lambda}$ clearly implies $\mathcal{A}_{\kappa, \lambda}^{\lambda^{+}}$, but the converse need not hold:

Proposition 7.6. Suppose $\mathrm{cf}(\lambda)<\kappa$ and there is a $\mathrm{cf}(\lambda)$-saturated ideal on $P_{\kappa}(\lambda)$. Then $\mathrm{ADS}_{\lambda}$ does not hold. 
Proof. Let $\left\langle a_{\alpha}: \alpha<\lambda^{+}\right\rangle$be as in the definition of $\operatorname{ADS}_{\lambda}$. For $\beta<\lambda^{+}$, pick $h_{\beta} \in \prod_{\alpha<\beta} a_{\alpha}$ so that the sequence $\left\langle a_{\alpha} \backslash h_{\beta}(\alpha): \alpha<\beta\right\rangle$ consists of pairwise disjoint sets. By Proposition 6.5 and Lemmas 1.4 and 6.7 there is a $\operatorname{cf}(\lambda)$-saturated ideal $J$ on $P_{\kappa}\left(\lambda^{+}\right)$. Given $\alpha<\lambda^{+}$, set

$$
A_{\alpha}^{\xi}=\left\{x \in P_{\kappa}\left(\lambda^{+}\right): \alpha<\bigcup x \text { and } h_{\bigcup x}(\alpha)=\xi\right\}
$$

for all $\xi \in a_{\alpha}$. There must be $e_{\alpha} \in P_{\operatorname{cf}(\lambda)}\left(a_{\alpha}\right)$ such that $\bigcup_{\xi \in e_{\alpha}} A_{\alpha}^{\xi} \in J^{*}$. Put $\eta_{\alpha}=\bigcup e_{\alpha}$. Now let $\alpha<\gamma<\lambda^{+}$. Pick $x \in\left(\bigcup_{\xi \in e_{\alpha}} A_{\alpha}^{\xi}\right) \cap\left(\bigcup_{\zeta \in e_{\gamma}} A_{\gamma}^{\zeta}\right)$. Then for $\sigma \in\{\alpha, \gamma\}, a_{\sigma} \backslash \eta_{\sigma} \subseteq a_{\sigma} \backslash h_{\cup x}(\sigma)$, and therefore $\left(a_{\alpha} \backslash \eta_{\alpha}\right) \cap\left(a_{\gamma} \backslash \eta_{\gamma}\right)=\emptyset$. Contradiction.

8. The square brackets partition relation on $P_{\kappa}(\lambda)$. Let $\kappa$ be a regular uncountable cardinal with the tree property. It is known [21] that if (a) $\left\{P_{\kappa}(\lambda)\right\} \rightarrow\left[I_{\kappa, \lambda}^{+}\right]_{\lambda<\kappa}^{2}$ for any cardinal $\lambda \geq \kappa$, and (b) $\kappa$ is inaccessible, then $\kappa$ is strongly compact. As will be shown below, (a) does not imply (b). In fact, if $\kappa$ Cohen reals are added to a model where $\kappa$ is supercompact, then in the generic extension $\left\{P_{\kappa}(\lambda)\right\} \rightarrow\left[I_{\kappa, \lambda}^{+}\right]_{\omega_{1}}^{2}$ for every $\lambda \geq \kappa$. We first establish a two-cardinal version of Lemma 7.4 (ii).

Throughout the remainder of this section $\kappa$ and $\lambda$ will denote two uncountable cardinals such that $\operatorname{cf}(\kappa)=\kappa \leq \lambda$.

The following is a straightforward generalization of a result of Solovay [32].

Lemma 8.1. Let $\tau$ and $\nu$ be two cardinals with $\omega_{1} \leq \tau \leq \nu<\kappa$, and $J$ be a $\tau$-saturated ideal on $P_{\kappa}(\lambda)$. Further, let $g: P_{\kappa}(\lambda) \times P_{\kappa}(\lambda) \rightarrow \nu$. Then one can find $E \in J^{*}$ and $e \in P_{\tau}(\nu)$ so that for any $a \in E,\left\{b \in P_{\kappa}(\lambda)\right.$ : $g(a, b) \in e\} \in J^{*}$.

Lemma 8.2. Let $J$ be a normal $\kappa$-saturated on $P_{\kappa}(\lambda)$. Further, let $A \in J^{+}$ and $g: A \rightarrow P_{\kappa}(\lambda)$ be such that $g(a) \in P_{|a \cap \kappa|}(a)$ for every $a \in A$. Then one can find $D \in J^{+} \cap P(A)$ and $x \in P_{\kappa}(\lambda)$ such that $g^{\prime \prime} D \subseteq P(x)$.

Proof. There must be $B \in J^{+} \cap P(A)$ and $\sigma<\kappa$ such that $|g(a)|=\sigma$ for every $a \in B$. For $a \in B$, let $g(a)=\left\{\gamma_{i}^{a}: i<\sigma\right\}$. For $i<\sigma$ and $\delta<\lambda$, set $B_{i}^{\delta}=\left\{a \in B: \gamma_{i}^{a}=\delta\right\}$. Clearly for every $i<\sigma$ and every $E \in J^{+} \cap P(B)$, there is $\delta<\lambda$ such that $E \cap B_{i}^{\delta} \in J^{+}$. Hence for each $i<\sigma$, one can find $e_{i} \in P_{\kappa}(\lambda)$ so that $W_{i} \in J^{*}$, where $W_{i}=\left(P_{\kappa}(\lambda) \backslash A\right) \cup \bigcup_{\delta \in e_{i}} B_{i}^{\delta}$. Put $C=\bigcap_{i<\sigma} W_{i}$ and $x=\bigcup_{i<\sigma} e_{i}$. Then $g(a) \subseteq x$ for every $a \in B \cap C$.

LEMma 8.3. Let $\chi>\lambda$ be a cardinal such that $\mathcal{A}_{\kappa, \lambda}^{\chi}$ holds, and $f$ : $P_{\kappa}(\lambda) \rightarrow P_{\kappa}(\chi)$ be the function defined in the proof of Lemma 6.4. Then for any normal $\kappa$-saturated ideal $J$ on $P_{\kappa}(\lambda), f(J)$ is a normal ideal on $P_{\kappa}(\chi)$.

Proof. Let $J$ be a normal $\kappa$-saturated ideal on $P_{\kappa}(\lambda)$. Set $H=f(J)$. Fix $X \in H^{+}$and $h: X \rightarrow \lambda^{+}$such that $h(x) \in x$ for all $x \in X$. Set 
$X_{0}=\{x \in X: h(x)<\lambda\}$ and $X_{1}=X \backslash X_{0}$. Pick $i \in\{0,1\}$ so that $X_{i} \in H^{+}$.

Case $i=0$ : There must be $A \in J^{+} \cap P\left(f^{-1}\left(X_{0}\right)\right)$ such that $h \circ f$ is constant on $A$. Set $Y=f^{\prime \prime} A$. Then $Y \in H^{+} \cap P\left(X_{0}\right)$ and $h$ is constant on $Y$.

Case $i=1$ : Define $g: f^{-1}\left(X_{1}\right) \rightarrow\left\{z_{\alpha}: \lambda \leq \alpha<\chi\right\}$ by $g(a)=z_{(h \circ f)(a)}$. Then by Lemma 8.2 there are $B \in J^{+} \cap P\left(f^{-1}\left(X_{1}\right)\right)$ and $e \in P_{\kappa}(\lambda)$ such that $g^{\prime \prime} B \subseteq P(e)$. Since $\left|\left\{\alpha: z_{\alpha} \subseteq e\right\}\right|<\kappa$, there must be $C \in J^{+} \cap P(B)$ such that $g$ is constant on $C$. Set $T=f^{\prime \prime} C$. Then $T \in H^{+} \cap P\left(X_{1}\right)$ and moreover $h$ is constant on $T$.

Proposition 8.4. Let $\tau$ and $\nu$ be two cardinals with $\omega_{1} \leq \tau \leq \nu<\kappa$, and $J$ be a seminormal $\tau$-saturated ideal on $P_{\kappa}(\lambda)$. Then $J^{+} \rightarrow\left[I_{\kappa, \lambda}^{+}\right]_{\nu,<\tau}^{2}$.

Proof. Case $\operatorname{cf}(\lambda) \geq \kappa$ : Fix $g: P_{\kappa}(\lambda) \times P_{\kappa}(\lambda) \rightarrow \nu$ and $A \in J^{+}$. By Lemma 8.1 one can find $E \in J^{*}$ and $e \in P_{\tau}(\nu)$ such that for any $a \in E$, $\left\{b \in P_{\kappa}(\lambda): g(a, b) \in e\right\} \in J^{*}$. By Proposition 6.3, there is $D \in I_{\kappa, \lambda}^{+}$with $|D|=\lambda$. Set $D=\left\{d_{\alpha}: \alpha<\lambda\right\}$. Inductively define $a_{\alpha} \in A \cap E$ for $\alpha<\lambda$ so that

(i) $d_{\alpha} \cup\{\alpha\} \subseteq a_{\alpha}$.

(ii) $a_{\alpha} \backslash a_{\beta} \neq 0$ for every $\beta \in \alpha$.

(iii) $g\left(a_{\beta}, a_{\alpha}\right) \in e$ for every $\beta \in \alpha \cap a_{\alpha}$.

Finally, let $C=\left\{a_{\alpha}: \alpha<\lambda\right\}$. Then clearly $C \in I_{\kappa, \lambda}^{+} \cap P(A)$. Moreover, if $\alpha, \beta \in \lambda$ are such that $a_{\beta} \subset a_{\alpha}$, then $\beta \in \alpha \cap a_{\alpha}$ and therefore $g\left(a_{\beta}, a_{\alpha}\right) \in e$.

Case $\operatorname{cf}(\lambda)<\kappa$ : By Lemmas 6.7 and $6.9, \mathcal{A}_{\kappa, \lambda}^{\lambda^{+}}$holds, so by Lemmas 1.2 and 8.3 there is $f: P_{\kappa}(\lambda) \rightarrow P_{\kappa}\left(\lambda^{+}\right)$such that (a) $f$ is an isomorphism from $\left(P_{\kappa}(\lambda), \subset\right)$ onto $(\operatorname{ran}(f), \subset)$, and (b) $f(J)$ is a normal ideal on $P_{\kappa}\left(\lambda^{+}\right)$. Now fix $A \in J^{+}$and $g: P_{\kappa}(\lambda) \times P_{\kappa}(\lambda) \rightarrow \nu$. Define $k: P_{\kappa}\left(\lambda^{+}\right) \times P_{\kappa}\left(\lambda^{+}\right) \rightarrow \nu$ so that for any $(a, b) \in P_{\kappa}(\lambda) \times P_{\kappa}(\lambda), k(f(a), f(b))=g(a, b)$. By the first part of the proof there are $X \in(f(J))^{+} \cap P\left(f^{\prime \prime} A\right)$ and $e \in P_{\tau}(\nu)$ such that $k(x, y) \in e$ for every $(x, y) \in X \times X$ with $x \subset y$. Set $B=f^{-1}(X)$. Then $B \in J^{+} \cap P(A)$ and $g(a, b) \in e$ for every $(a, b) \in B \times B$ with $a \subset b$.

Proposition 8.5. Let $J$ be an ideal on $P_{\kappa}(\lambda)$ and $\nu<\kappa$ be an infinite cardinal. Further, let $P$ be a $\nu$-cc forcing notion and $G$ be $P$-generic over $V$. In $V[G]$, let $H$ be the set of all $X \subseteq P_{\kappa}(\lambda)$ such that $X \cap A=\emptyset$ for some $A \in J^{*}$. Then the following hold:

(i) Suppose $J$ is $\tau$-saturated, where $\tau$ is a regular uncountable cardinal with $\nu \leq \tau \leq \kappa^{+}$. Then $H$ is a $\tau$-saturated ideal on $P_{\kappa}(\lambda)$.

(ii) Suppose $J$ is prime and normal. Then $H$ is normal. In fact, for any $f: P_{\kappa}(\lambda) \rightarrow \lambda$ with $f(x) \in x$ for every $x \in P_{\kappa}(\lambda) \backslash\{\emptyset\}$, there are $X \in H^{*}$ and $e \in P_{\nu}(\lambda)$ such that $f(x) \in e$ for all $x \in X$. 
Proof. (i) This is a straightforward generalization of a result of Prikry, Solovay and Kakuda (see Theorem 17.1 in [14]).

(ii) This is proved by a standard argument (see e.g. the proof of Lemma 2.4 in [1]).

COROllary 8.6. Suppose $\kappa$ is $\lambda$-supercompact. Let $\tau<\kappa$ be a regular uncountable cardinal and $P$ be a $\tau$-cc forcing notion. Further, let $G$ be $P$ generic over $V$. Then in $V[G]$, there is a normal $\tau$-saturated ideal $H$ on $P_{\kappa}(\lambda)$.

9. Solovay's result on the sup-function. Throughout this section $\kappa$ and $\lambda$ denote two cardinals such that $\operatorname{cf}(\kappa)=\kappa \leq \lambda$.

Solovay [33] established that if $\lambda$ is a regular cardinal, $\kappa$ is $\lambda$-supercompact and $J$ is a normal prime ideal on $P_{\kappa}(\lambda)$, then the function $a \mapsto \bigcup a$ is one-to-one on a set in $J^{*}$. Assuming that $\lambda$ is a successor cardinal, Johnson [13] sharpened Solovay's result by proving that if $\kappa$ is $\lambda$-Shelah, then the supfunction is one-to-one on a set in $N S h_{\kappa, \lambda}^{*}$. Abe [3] observed that Johnson's result is still valid in the case when $\lambda$ is a regular limit cardinal.

Definition. $N S h_{\kappa, \lambda}$ is the set of all $A \subseteq P_{\kappa}(\lambda)$ for which one can find $g_{a}: a \rightarrow a$ for $a \in A$ so that for every $f: \lambda \rightarrow \lambda$, there is $b \in P_{\kappa}(\lambda)$ with $\left\{a \in A: b \subseteq a\right.$ and $f\left\lceil b=g_{a}\lceil b\}=\emptyset\right.$.

$\kappa$ is $\lambda$-Shelah if $P_{\kappa}(\lambda) \notin N S h_{\kappa, \lambda}$.

LEMMA 9.1.

(i) (Carr [4]) If $\kappa$ is $\lambda$-Shelah, then it is mildly $\lambda$-ineffable.

(ii) (Carr [4], Usuba [37]) If $\kappa$ is $\lambda$-Shelah, then $N S h_{\kappa, \lambda}$ is a strongly normal ideal on $P_{\kappa}(\lambda)$.

Lemma 9.2. Let $\mu$ be a cardinal with $\kappa \leq \mu \leq \lambda$. Then the following hold:

(i) (Essentially due to Johnson [13]) $\left\{a \in P_{\kappa}(\lambda)\right.$ : o.t. $(a \cap \mu)$ is not $a$ cardinal $\} \in N S h_{\kappa, \lambda}$.

(ii) (Abe [3]) $\left\{a \in P_{\kappa}(\lambda): \operatorname{cf}(|a \cap \mu|) \neq|a \cap \operatorname{cf}(\mu)|\right\} \in N S h_{\kappa, \lambda}$.

Proposition 9.3 (Abe [3]). Suppose that $\kappa$ is $\lambda$-Shelah and $\lambda$ is a regular cardinal. Then the function $a \mapsto \bigcup a$ is injective on a set in $N S h_{\kappa, \lambda}^{*}$.

Proof. The proof is similar to that of Theorem 2.1 in [13], using Lemma 9.2 instead of Lemma 2.4 in [13].

What if $\kappa$ is $\lambda$-Shelah and $\lambda$ is a singular cardinal? We first consider the case when $\operatorname{cf}(\lambda) \geq \kappa$.

Proposition 9.4. Suppose $\kappa \leq \operatorname{cf}(\lambda)<\lambda$ and $\kappa$ is $\lambda$-Shelah. Let $\left\langle\lambda_{i}\right.$ : $i<\operatorname{cf}(\lambda)\rangle$ be a strictly increasing sequence of regular cardinals such that $\lambda_{0}=\operatorname{cf}(\lambda)$ and $\bigcup_{i<\operatorname{cf}(\lambda)} \lambda_{i}=\lambda$. For $a \in P_{\kappa}(\lambda)$, define $k_{a} \in \prod_{i \in a \cap \operatorname{cf}(\lambda)} \lambda_{i}$ 
by $k_{a}(i)=\bigcup\left(a \cap \lambda_{i}\right)$. Then the function $a \mapsto k_{a}$ is one-to-one on a set in $N S h_{\kappa, \lambda}^{*}$.

Proof. Put $\sigma=\operatorname{cf}(\lambda)$. Let $A$ be the set of all $a \in P_{\kappa}(\lambda)$ such that (a) $\omega \subseteq a$, (b) $\left\{\lambda_{i}: i \in a \cap \sigma\right\} \subseteq a$, and (c) $\left\{i_{\alpha}: \alpha \in a\right\} \subseteq a$, where $i_{\alpha}=$ the least $i<\sigma$ such that $\alpha \in \lambda_{i}$. Then clearly $A \in N S_{\kappa, \lambda}^{*}$. Note that $\bigcup a=\bigcup_{i \in a \cap \sigma} \lambda_{i}$ for every $a \in A$. By Proposition 9.3 one can find $B_{i} \in N S h_{\kappa, \lambda_{i}}^{*}$ for $i<\sigma$ so that the function $b \mapsto \bigcup b$ is injective on $B_{i}$. For $i<\sigma$, set $C_{i}=\left\{a \in P_{\kappa}(\lambda): a \cap \lambda_{i} \in B_{i}\right\}$. It is simple to see that $C_{i} \in N S h_{\kappa, \lambda}^{*}$ for all $i$. Put $D=\left\{a \in A: \forall i \in a \cap \sigma\left(a \in C_{i}\right)\right\}$. Note that $D \in N S h_{\kappa, \lambda}^{*}$. Now fix $a, b \in D$ with $a \neq b$. If $a \cap \sigma \neq b \cap \sigma$, then $k_{a}(0) \neq k_{b}(0)$ (and therefore $\bigcup a \neq \bigcup b$ ). Otherwise, let $j=$ the least $k \in a \cap \sigma$ such that $a \cap \lambda_{k} \neq b \cap \lambda_{k}$. Then $k_{a}(i) \neq k_{b}(i)$ for any $i \in(a \cap \sigma) \backslash j$.

Note that in the statement of Proposition 9.4, the range of the function $a \mapsto k_{a}$ has size $\lambda$.

It remains to deal with the case $\operatorname{cf}(\lambda)<\kappa$.

Definition. Let $X$ be an infinite set. An $\omega$-Jónsson function for $X$ is a function $F:{ }^{\omega} X \rightarrow X$ such that $F^{\prime \prime}{ }^{\omega} Y=X$ for every $Y \subseteq X$ with $|Y|=|X|$.

\section{LEMMA 9.5.}

(i) (Erdős-Hajnal [8]) For any infinite set $X$, there exists an $\omega$-Jónsson function for $X$.

(ii) (Johnson [13]) Let $\mu$ be a cardinal with $\kappa \leq \mu \leq \lambda$, and $F$ be an $\omega$-Jónsson function for $\mu$. Then the set of all $a \in P_{\kappa}(\lambda)$ such that $\omega \subseteq a$ and $F \Gamma^{\omega}(a \cap \mu)$ is not an $\omega$-Jónsson function for $a \cap \mu$ lies in $N S h_{\kappa, \lambda}$.

Usuba [37] proved that if $\operatorname{cf}(\lambda)<\kappa$ and $\kappa$ is $\lambda$-Shelah, then $\lambda^{<\kappa}=\lambda^{+}$. The following is a refinement of this result.

Proposition 9.6. Suppose that $\operatorname{cf}(\lambda)<\kappa$ and $\kappa$ is $\lambda$-Shelah. Let $\left\langle\lambda_{i}\right.$ : $i<\operatorname{cf}(\lambda)\rangle$ be an increasing sequence of regular cardinals such that $\kappa \leq \lambda_{0}$ and $\bigcup_{i<\mathrm{cf}(\lambda)} \lambda_{i}=\lambda$, and let $\left\langle f_{\alpha}: \alpha<\lambda^{+}\right\rangle$be a scale on $\prod_{i<\mathrm{cf}(\lambda)} \lambda_{i}$. For $a \in P_{\kappa}(\lambda)$, define $k_{a} \in \prod_{i<\operatorname{cf}(\lambda)} \lambda_{i}$ by $k_{a}(i)=\bigcup\left(a \cap \lambda_{i}\right)$. Then the function $a \mapsto$ the least $\alpha$ such that $\neg\left(f_{\alpha}<^{*} k_{a}\right)$ is injective on a set in $N S h_{\kappa, \lambda}^{*}$.

Proof. For $\gamma<\lambda$, put $z_{\gamma}=\{\gamma\}$. Inductively define $z_{\beta} \in P_{\kappa}(\lambda)$ for $\lambda \leq \beta<\lambda^{+}$so that $z_{\beta} \notin\left\{z_{\delta}: \delta<\beta\right\}$. Define $t: P_{\kappa}(\lambda) \rightarrow P_{\kappa}\left(\lambda^{+}\right)$by $t(a)=\left\{\alpha<\lambda^{+}: z_{\alpha} \in P_{|a \cap \kappa|}(a)\right\}$. For $i<\operatorname{cf}(\lambda)$, pick an $\omega$-Jónsson function $F_{i}$ for $\lambda_{i}$. Now let $A$ be the set of all $a \in P_{\kappa}(\lambda)$ such that

(1) $a \cap \kappa$ is an inaccessible cardinal.

(2) $a \cap \kappa>\operatorname{cf}(\lambda)$. 
(3) For each $i<\operatorname{cf}(\lambda)$, o.t. $\left(a \cap \lambda_{i}\right)$ is a regular cardinal and $\left.F_{i}\right|^{\omega}\left(a \cap \lambda_{i}\right)$ is an $\omega$-Jónsson function for $a \cap \lambda_{i}$.

(4) Let $\alpha<\lambda^{+}$be such that $z_{\alpha} \in P_{a \cap \kappa}(a)$. Then (a) $z_{\alpha+1} \in P_{a \cap \kappa}(a)$, (b) $\operatorname{ran}\left(f_{\alpha}\right) \subseteq a$, and (c) $z_{\bigcup d} \in P_{a \cap \kappa}(a)$ for every $d \subseteq\left\{\delta<\lambda^{+}\right.$: $\left.z_{\delta} \subseteq z_{\alpha}\right\}$.

(5) For any $g \in \prod_{i<\mathrm{cf}(\lambda)}\left(a \cap \lambda_{i}\right)$, there is $\delta<\lambda^{+}$such that $g<^{*} f_{\delta}$ and $z_{\delta} \in P_{a \cap \kappa}(a)$.

It is simple to see that $A \in N S h_{\kappa, \lambda}^{*}$. Note that $\lambda \cap t(a)=a$ for every $a \in A$. Define $h: A \rightarrow \lambda^{+}$by $h(a)=\bigcup t(a)$. Let us first show that $h$ is one-to-one. Thus let $a_{0}, a_{1} \in A$ with $h\left(a_{0}\right)=h\left(a_{1}\right)$. Put $x=t\left(a_{0}\right) \cap t\left(a_{1}\right)$ and $\sigma=h\left(a_{0}\right)=h\left(a_{1}\right)$.

Claim 1. Let $\delta<\sigma$. Then there is $\alpha \in x$ with $\delta<\alpha$.

Proof of Claim 1. Inductively define $\alpha_{j}$ for $j<\omega$ so that (a) $\alpha_{2 j} \in$ $t\left(a_{0}\right)$ and $\alpha_{2 j+1} \in t\left(a_{1}\right)$, and (b) $\delta<\alpha_{0}<\alpha_{1}<\alpha_{2}<\cdots$. Then clearly $\bigcup_{j \in \omega} z_{\alpha_{2 j}} \in P_{a_{0} \cap \kappa}\left(a_{0}\right)$ and $\bigcup_{j \in \omega} z_{\alpha_{2 j+1}} \in P_{a_{1} \cap \kappa}\left(a_{1}\right)$. It follows that $\bigcup_{j \in \omega} \alpha_{j}$ $\in x$, which completes the proof of the claim.

Claim 2. Let $k<2$. Then $\left|\left\{i<\operatorname{cf}(\lambda): \bigcup\left(x \cap \lambda_{i}\right)<\bigcup\left(a_{k} \cap \lambda_{i}\right)\right\}\right|<\operatorname{cf}(\lambda)$.

Proof of Claim 2. Suppose otherwise. Let

$$
T=\left\{i<\operatorname{cf}(\lambda): \bigcup\left(x \cap \lambda_{i}\right)<\bigcup\left(a_{k} \cap \lambda_{i}\right)\right\} .
$$

Define $g \in \prod_{i<\operatorname{cf}(\lambda)}\left(a_{k} \cap \lambda_{i}\right)$ by: $g(i) \in\left(a_{k} \cap \lambda_{i}\right) \backslash \bigcup\left(x \cap \lambda_{i}\right)$ if $i \in T$, and $g(i)=0$ otherwise. There must be $\delta \in t\left(a_{k}\right)$ such that $g<^{*} f_{\delta}$. By Claim 1, there is $\alpha \in x$ with $\delta<\alpha$. Then $\operatorname{ran}\left(f_{\alpha}\right) \subseteq a_{0} \cap a_{1} \subseteq x$. But $f_{\delta}<^{*} f_{\alpha}$, so there is $i \in T$ such that $f_{\alpha}(i)>g(i)$. This contradiction completes the proof of Claim 2.

Claim 3. Let $k<2$. Then $a_{k}=x \cap \lambda$.

Proof of Claim 3. Let $i<\operatorname{cf}(\lambda)$ be such that $\bigcup\left(x \cap \lambda_{i}\right)=\bigcup\left(a_{k} \cap \lambda_{i}\right)$. Then o.t. $\left(x \cap \lambda_{i}\right)=$ o.t. $\left(a_{k} \cap \lambda_{i}\right)$ since o.t. $\left(a_{k} \cap \lambda_{i}\right)$ is a regular cardinal. Moreover, $F_{i}^{\prime \prime}{ }^{\omega}\left(x \cap \lambda_{i}\right) \subseteq x \cap \lambda_{i}$, and consequently $x \cap \lambda_{i}=a_{k} \cap \lambda_{i}$. The desired conclusion is now immediate from Claim 2.

By Claim 3, $a_{0}=a_{1}$, which completes our proof of the injectivity of $h$. Let us finally prove that for any $a \in A, h(a)=$ the least $\alpha$ such that $\neg\left(f_{\alpha}<^{*} k_{a}\right)$. Thus fix $a \in A$. We show that $h(a)=\left\{\beta<\lambda^{+}: f_{\beta}<^{*} k_{a}\right\}$. Firstly, let $\beta<\lambda^{+}$with $f_{\beta}<^{*} k_{a}$. We may find $g \in \prod_{i<\operatorname{cf}(\lambda)}\left(a \cap \lambda_{i}\right)$ such that $\left|\left\{i<\operatorname{cf}(\lambda): f_{\beta}(i)>g(i)\right\}\right|<\operatorname{cf}(\lambda)$. There is $\delta \in t(a)$ such that $g<^{*} f_{\delta}$. Then clearly $\beta<\delta$, so $\beta<h(a)$. Conversely, suppose $\zeta<h(a)$. Pick $\xi \in t(a)$ with $\zeta<\xi$. Since $\operatorname{ran}\left(f_{\xi}\right) \subseteq a, f_{\zeta}<^{*} f_{\xi}<^{*} k_{a}$ and hence $f_{\zeta}<k_{a}$. 
10. $S_{\kappa, \lambda}$. Throughout this section $\kappa$ and $\lambda$ will denote two uncountable cardinals such that $\operatorname{cf}(\kappa)=\kappa<\lambda$.

Definition. $S_{\kappa, \lambda}=\left\{a \in P_{\kappa}(\lambda):|a|=|a \cap \kappa|\right\}$.

Krueger [15] showed that it is consistent (relative to a supercompact cardinal) that "there is a supercompact cardinal $\tau$ such that

$$
\left\{a \in P_{\tau}\left(\tau^{+}\right):|a \cap \tau| \text { is not measurable }\right\} \backslash S_{\tau, \tau^{+}} \in N S_{\tau, \tau^{+}} " .
$$

Moreover, he showed that it is consistent (relative to the same assumption) that "there is an uncountable strongly compact cardinal $\tau$ such that $P_{\tau}\left(\tau^{+}\right) \backslash S_{\tau, \tau^{+}} \in N S_{\tau, \tau^{+}}$". We will show that these results are optimal in the sense that if $\kappa$ is $\kappa^{+}$-compact, then

$$
\left\{a \in P_{\kappa}\left(\kappa^{+}\right):|a \cap \kappa| \text { is not measurable }\right\} \backslash S_{\kappa, \kappa^{+}} \in N S S_{\kappa, \kappa^{+}}^{+} .
$$

Definition. Let $0<\delta \leq \lambda$. Then $N S h_{\kappa, \lambda}^{\delta}$ denotes the set of all $B \subseteq$ $P_{\kappa}(\lambda)$ such that one can find $h_{b}: b \rightarrow b \cap \delta$ for $b \in B$ with $b \cap \delta \neq \emptyset$ so that for any $u: \lambda \rightarrow \delta$, there is $a \in P_{\kappa}(\lambda)$ with $h_{b}\lceil a \neq u\lceil a$ for all $b \in B$ such that $a \subseteq b$ and $b \cap \delta \neq \emptyset$.

Note that $N S h_{\kappa, \lambda}^{\lambda}=N S h_{\kappa, \lambda}$.

LEMMA 10.1.

(i) $N S h_{\kappa, \lambda}^{1}=I_{\kappa, \lambda}$.

(ii) Let $0<\eta<\delta \leq \lambda$. Then $N S h_{\kappa, \lambda}^{\eta} \subseteq N S h_{\kappa, \lambda}^{\delta}$.

(iii) $P_{\kappa}(\lambda) \notin N S h_{\kappa, \lambda}^{2}$ if and only if $\kappa$ is mildly $\lambda$-ineffable.

(iv) (Carr [4]) If $P_{\kappa}(\lambda) \notin N S h_{\kappa, \lambda}^{2}$, then $N S h_{\kappa, \lambda}^{2}=I_{\kappa, \lambda}$.

(v) Let $2<\delta<\kappa$. Then $N S h_{\kappa, \lambda}^{\delta}=N S h_{\kappa, \lambda}^{2}$.

Recall that if $\kappa$ is $\lambda$-Shelah, then $N S h_{\kappa, \lambda}^{\lambda}$ is a normal ideal on $P_{\kappa}(\lambda)$. This can be generalized as follows.

Lemma 10.2. Let $\kappa \leq \delta \leq \lambda$. Suppose $P_{\kappa}(\lambda) \notin N S h_{\kappa, \lambda}^{\delta}$. Then $N S h_{\kappa, \lambda}^{\delta}$ is a $\delta$-normal ideal on $P_{\kappa}(\lambda)$.

Proof. It is simple to see that $P(B) \subseteq N S h_{\kappa, \lambda}^{\delta}$ for every $B \in N S h_{\kappa, \lambda}^{\delta}$.

Claim. Suppose $B_{\gamma} \in N S h_{\kappa, \lambda}^{\delta}$ for $\gamma<\delta$, and let $C$ be the set of all $c \in \bigcup_{\gamma<\delta}\left\{b \in B_{\gamma}: \gamma \in b\right\}$ such that $\omega \subseteq c$. Then $C \in N S h_{\kappa, \lambda}^{\delta}$.

Proof of the claim. Pick $f: C \rightarrow \delta$ so that for any $b \in C, f(b) \in b \in$ $B_{f(b)}$. For $\gamma<\delta$, select $h_{b}^{\gamma}: b \rightarrow b \cap \delta$ for $b \in B_{\gamma}$ with $b \cap \delta \neq \emptyset$ so that for any $u: \lambda \rightarrow \delta$, there is $a \in P_{\kappa}(\lambda)$ with $h_{b}^{\gamma}\left\lceil a \neq u\left\lceil a\right.\right.$ for all $b \in B_{\gamma}$ such that $a \subseteq b$ and $b \cap \delta \neq \emptyset$. For $b \in C$, define $k_{b}: b \rightarrow b \cap \delta$ by $k_{b}(0)=f(b)$ and $k_{b}(1+\zeta)=h_{b}^{f(b)}(\zeta)$. Now assume there is $t: \lambda \rightarrow \delta$ with the property that for any $d \in P_{\kappa}(\lambda)$, there is $b \in C$ such that $d \subseteq b$ and $k_{b}\lceil d=t\lceil d$. Define $u: \lambda \rightarrow \delta$ by $u(\zeta)=t(1+\zeta)$. Given $a \in P_{\kappa}(\lambda)$, put $d=a \cup \omega$ and select $b \in C$ 
so that $d \subseteq b$ and $k_{b}\left\lceil d=t\left\lceil d\right.\right.$. Then clearly $a \subseteq b$ and $b \in B_{t(0)}$. Moreover, for each $\zeta \in a, u(\zeta)=t(1+\zeta)=k_{b}(1+\zeta)=h_{b}^{t(0)}(\zeta)$. This contradiction completes the proof of the claim.

Now let $A \in P\left(P_{\kappa}(\lambda)\right) \backslash N S h_{\kappa, \lambda}^{\delta}$ and $g: A \rightarrow \delta$ be such that $g(a) \in a \cap \delta$ for every $a \in A$. Since

$$
\left\{a \in \bigcup_{\gamma<\delta} g^{-1}(\{\gamma\}): \omega \subseteq a\right\} \notin N S h_{\kappa, \lambda}^{\delta},
$$

it follows from the claim that $g^{-1}(\{\gamma\}) \notin N S h_{\kappa, \lambda}^{\delta}$ for some $\gamma<\delta$.

Lemma 10.3. Let $\kappa \leq \delta<\lambda$. Then $\left\{b \in P_{\kappa}(\lambda):|b|=|b \cap \delta|\right\} \in N S h_{\kappa, \lambda}^{\delta}$.

Proof. Suppose to the contrary that $B \notin N S h_{\kappa, \lambda}^{\delta}$, where $B=\{b \in$ $\left.P_{\kappa}(\lambda):|b|=|b \cap \delta|\right\}$. For $b \in B$, select a one-to-one function $h_{b}: b \rightarrow b \cap \delta$. There must be $u: \lambda \rightarrow \delta$ such that for any $a \in P_{\kappa}(\lambda)$, there is $b \in B$ such that $a \subseteq b$ and $h_{b}\lceil a=u\lceil a$. Then $u$ is one-to-one, a clear contradiction.

Proposition 10.4. Suppose $\lambda=\kappa^{+}$and $P_{\kappa}(\lambda) \notin \bigcup_{\kappa \leq \delta<\kappa^{+}} N S h_{\kappa, \lambda}^{\delta}$. Then $P_{\kappa}(\lambda) \backslash S_{\kappa, \lambda} \in N S S_{\kappa, \lambda}^{+}$.

Proof. Since clearly $N S S_{\kappa, \lambda}=\bigcup_{\kappa \leq \delta<\lambda} N S_{\kappa, \lambda}^{\delta}$, the result follows from Lemmas 10.2 and 10.3 .

Lemma 10.5. Let $\kappa \leq \delta \leq \lambda$. Suppose $H$ is a $\delta$-normal prime ideal on $P_{\kappa}(\lambda)$. Then $N S h_{\kappa, \lambda}^{\delta} \subseteq H$.

Proof. Let $B \in H^{+}$, and let $h_{b}: b \rightarrow b \cap \delta$ for $\delta \in B^{\prime}$, where $B^{\prime}=\{b \in B$ : $0 \in b\}$. Define $u: \lambda \rightarrow \delta$ so that for any $\alpha \in \lambda$,

$$
X_{\alpha}=\left\{b \in B^{\prime}: h_{b}(\alpha)=u(\alpha)\right\}
$$

lies in $H^{+}$. Now given $a \in P_{\kappa}(\lambda)$, pick $b \in \bigcap_{\alpha \in a} X_{\alpha}$ with $a \subseteq b$. Then clearly $h_{b}\left\lceil a=u\left\lceil a\right.\right.$. Hence $B \notin N S h_{\kappa, \lambda}^{\delta}$.

Lemma 10.6 (Matet [19]). Suppose $\kappa$ is $\lambda$-compact. Let $\kappa \leq \delta<\kappa^{+}$, and let $J$ be a normal prime ideal on $\kappa$. Then there is a $\delta$-normal prime ideal $H$ on $P_{\kappa}(\lambda)$ such that (a) $J=\left\{D \subseteq \kappa:\left\{a \in P_{\kappa}(\lambda): \bigcup(a \cap \kappa) \in D\right\} \in H\right\}$, and (b) $\left\{a \in P_{\kappa}(\lambda):|a| \leq f(\bigcup(a \cap \kappa))\right\} \in H$ for every $f: \kappa \rightarrow \kappa$.

LEMma 10.7 (Solovay, see [14, p. 55]). If $\kappa$ is a measurable cardinal, then there exists a normal prime ideal $J$ on $\kappa$ such that

$$
\{\rho<\kappa: \rho \text { is a measurable cardinal }\} \in J .
$$

Proposition 10.8. Suppose $\kappa$ is $\lambda$-compact. Then for any $f: \kappa \rightarrow \kappa$, $A_{f} \notin \bigcup_{\kappa \leq \delta<\kappa^{+}} N S h_{\kappa, \lambda}^{\delta}$, where $A_{f}$ denotes the set of all $a \in P_{\kappa}(\lambda)$ such that (a) $a \cap \kappa$ is a nonmeasurable infinite cardinal, and (b) $|a|>f(a \cap \kappa)$. 


\section{Proof. By Lemmas 10.5-10.7.}

To conclude let us remark that a modification of the proof of Lemma 4.2 yields the following.

Proposition 10.9. Let $\mu$ be a cardinal with $\kappa \leq \mu<\lambda$. Then

$$
\overline{\operatorname{cof}}\left(N S_{\kappa, \lambda}^{\mu} \mid S_{\kappa, \lambda}\right) \geq \operatorname{cov}\left(\lambda, \mu^{+}, \mu^{+}, \kappa\right) .
$$

Proof. Select a family $\mathcal{G}$ of functions from $P_{\omega}(\mu)$ to $P_{\kappa}(\lambda)$ so that $|\mathcal{G}|=$ $\overline{\operatorname{cof}}\left(N S_{\kappa, \lambda}^{\mu} \mid S_{\kappa, \lambda}\right)$ and for any $h: P_{\omega}(\mu) \rightarrow P_{\kappa}(\lambda)$, there is $X \in P_{\kappa}(\mathcal{G}) \backslash\{\emptyset\}$ with $S_{\kappa, \lambda} \cap \bigcap_{g \in X} C_{g}^{\kappa, \lambda} \subseteq C_{h}^{\kappa, \lambda}$. For $g \in \mathcal{G}$, let $B_{g}=\mu \cup \bigcup \operatorname{ran}(g)$. Now fix $D \subseteq \lambda$ with $|D| \leq \mu$. Pick $h: P_{\omega}(\mu) \rightarrow P_{\kappa}(\lambda)$ and $X \in P_{\kappa}(\mathcal{G}) \backslash\{\emptyset\}$ so that $D \subseteq \cup \operatorname{ran}(h)$ and $S_{\kappa, \lambda} \cap \bigcap_{g \in X} C_{g}^{\kappa, \lambda} \subseteq C_{h}^{\kappa, \lambda}$. For $e \in P_{\omega}(\mu)$, define inductively $s_{n}^{e}$ for $n<\omega$ by $s_{0}^{e}=e \cup \omega$ and

$$
s_{n+1}^{e}=s_{n}^{e} \cup\left|s_{n}^{e}\right| \cup \bigcup\left\{g(d): g \in X \text { and } d \in P_{\omega}\left(s_{n}^{e} \cap \mu\right)\right\} .
$$

Let $z_{e}=\bigcup_{n<\omega} s_{n}^{e}$. Then $z_{e}$ belongs to $S_{\kappa, \lambda} \cap \bigcap_{g \in X} C_{g}^{\kappa, \lambda}$ and therefore to $C_{h}^{\kappa, \lambda}$. Hence,

$$
D \subseteq \bigcup\left\{z_{e}: e \in P_{\omega}(\mu)\right\} \subseteq \bigcup\left\{B_{g}: g \in X\right\} .
$$

Question. Is it true that $\overline{\operatorname{cof}}\left(N S_{\kappa, \lambda}^{\mu} \mid S_{\kappa, \lambda}\right)=\overline{\operatorname{cof}}\left(N S_{\kappa, \lambda}^{\mu}\right)$ for every cardinal $\mu$ with $\kappa \leq \mu<\lambda$ ?

Acknowledgements. The author wishes to thank Stevo Todorcevic for stimulating discussions on the subject matter of this paper.

\section{References}

[1] Y. Abe, Weakly normal filters and large cardinals, Tsukuba J. Math. 16 (1992), 487-494.

[2] - Weakly normal ideals on $\mathcal{P}_{\kappa} \lambda$ and the singular cardinal hypothesis, Fund. Math. 143 (1993), 97-106.

[3] -, Combinatorial characterization of $\Pi_{1}^{1}$-indescribability in $P_{\kappa} \lambda$, Arch. Math. Logic 37 (1998), 261-272.

[4] D. M. Carr, The structure of ineffability properties of $P_{\kappa} \lambda$, Acta Math. Hungar. 47 (1986), 325-332.

[5] D. M. Carr, J. P. Levinski and D. H. Pelletier, On the existence of strongly normal ideals over $P_{\kappa} \lambda$, Arch. Math. Logic 30 (1990), 59-72.

[6] C. A. Di Prisco and W. S. Zwicker, Flipping properties and supercompact cardinals, Fund. Math. 109 (1980), 31-36.

[7] H. D. Donder and P. Matet, Two cardinal versions of diamond, Israel J. Math. 83 (1993), 1-43.

[8] P. Erdős and A. Hajnal, On a problem of B. Jónsson, Bull. Acad. Polon. Sci. Sér. Sci. Math. Astronom. Phys. 14 (1966), 19-23.

[9] P. Erdős and A. Tarski, On families of mutually exclusive sets, Ann. of Math. 44 (1943), 315-329. 
[10] M. Gitik, The strength of the failure of the Singular Cardinal Hypothesis, Ann. Pure Appl. Logic 51 (1991), 216-240.

[11] M. Gitik and S. Shelah, On certain indestructibility of strong cardinals and a question of Hajnal, Arch. Math. Logic 28 (1989), 35-42.

[12] M. Holz, K. Steffens and E. Weitz, Introduction to Cardinal Arithmetic, Birkhäuser, Basel, 1999.

[13] C. A. Johnson, Some partition relations for ideals on $P_{\kappa} \lambda$, Acta Math. Hungar. 56 (1990), 269-282.

[14] A. Kanamori, The Higher Infinite, Perspectives in Math. Logic, Springer, Berlin, 1994.

[15] J. Krueger, Strong compactness and stationary sets, J. Symbolic Logic 70 (2005), 767-777.

[16] K. Kunen, Some applications of iterated ultrapowers in set theory, Ann. Math. Logic 1 (1970), 179-227.

[17] A. Liu, Bounds for covering numbers, J. Symbolic Logic 71 (2006), 1303-1310.

[18] M. Magidor and S. Shelah, When does almost free imply free? (for groups, transversals, etc.), J. Amer. Math. Soc. 7 (1994), 769-830.

[19] P. Matet, A partition property of a mixed type for $P_{\kappa}(\lambda)$, Math. Logic Quart. 49 (2003), 1-14.

[20] - Covering for category and combinatorics on $P_{\kappa}(\lambda)$, J. Math. Soc. Japan 58 (2006), 153-181.

[21] —, Strong compactness and a partition property, Proc. Amer. Math. Soc. 134 (2006), $2147-2152$.

[22] - Weak saturation of ideals on $P_{\kappa}(\lambda)$, preprint.

[23] P. Matet, C. Péan and S. Shelah, Cofinality of normal ideals on $P_{\kappa}(\lambda) I$, preprint (http://archiv.org//abs/math/0404318).

[24] - - - - Cofinality of normal ideals on $P_{\kappa}(\lambda)$ II, Israel J. Math. 150 (2005), $253-283$.

[25] Y. Matsubara, Menas' conjecture and generic ultrapowers, Ann. Pure Appl. Logic 36 (1987), 225-234.

[26] A. Rinot, On the consistency strength of the Milner-Sauer conjecture, ibid. 140 (2006), 110-119.

[27] S. Shelah, Advances in cardinal arithmetic, in: Finite and Infinite Combinatorics in Sets and Logic, B. Sands et al. (eds.), NATO ASI Ser. C 411, Kluwer, Dordrecht, 1993, 355-383.

[28] —, Cardinal Arithmetic, Oxford Logic Guides 29, Oxford Univ. Press, Oxford, 1994.

[29] —, Further cardinal arithmetic, Israel J. Math. 95 (1996), 61-114.

[30] —, The Generalized Continuum Hypothesis revisited, ibid. 116 (2000), 285-321.

[31] J. H. Silver, On the singular cardinals problem, in: Proc. Int. Congress Math., Vancouver 1974, Vol. I, 265-268.

[32] R. M. Solovay, Real-valued measurable cardinals, in: Axiomatic Set Theory, D. S. Scott (ed.), Proc. Sympos. Pure Math. 13, Part 1, Amer. Math. Soc., Providence, 1971, 397-428.

[33] - Strongly compact cardinals and the GCH, in: Proceedings of the Tarski Symposium, L. Henkin et al. (eds.), Proc. Sympos. Pure Math. 25, Amer. Math. Soc., Providence, 1974, 365-372.

[34] S. Todorčević, On a conjecture of R. Rado, J. London Math. Soc. 27 (1983), 1-8.

[35] - Conjectures of Rado and Chang and cardinal arithmetic, in: Finite and Infinite Combinatorics in Sets and Logic, B. Sands et al. (eds.), NATO ASI Ser. C 411, Kluwer, Dordrecht, 1993, 385-398. 
[36] S. Ulam, Zur Masstheorie in der allgemeinen Mengenlehre, Fund. Math. 16 (1930), 140-150.

[37] T. Usuba, Ineffability of $\mathcal{P}_{\kappa} \lambda$ for $\lambda$ with small cofinality, J. Math. Soc. Japan 60 (2008), 935-954.

[38] - Splitting stationary sets in $\mathcal{P}_{\kappa} \lambda$ for $\lambda$ with small cofinality, preprint.

CNRS Laboratoire de Mathématiques

Université de Caen

BP 5186

14032 Caen Cedex, France

E-mail: matet@math.unicaen.fr

Received 6 July 2007;

in revised form 22 May 2009 